\title{
TIDAL VELOCITY ASYMMETRIES AND \\ BEDLOAD TRANSPORT \\ IN SHALLOW EMBAYMENTS
}

by

Virginia Ann Fry

B.S., Pennsylvania State University, University Park, PA

(1981)

\section{SUBMITTED TO THE DEPARTMENT OF EARTH, ATMOSPHERIC AND PLANETARY SCIENCES \\ IN PARTIAL FULFILLMENT \\ OF THE REQUIREMENTS FOR THE \\ DEGREE OF}

\section{MASTER OF SCIENCE IN OCEANOGRAPHY}

at the

\section{MASSACHUSETTS INSTITUTE OF TECHNOLOGY}

August 1987

(C)Virginia A. Fry 1987

The author hereby grants to M.I.T. permission to reproduce and to distribute copies of this thesis document in whple or in part.

Signature of Author -

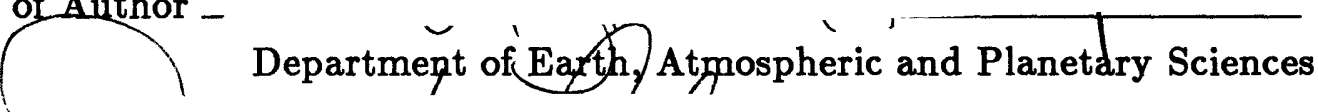

Certified by

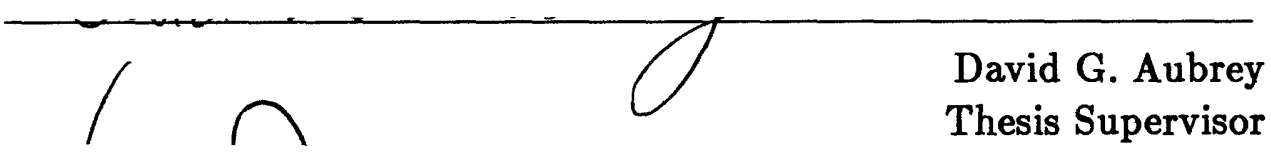

Accepted by

Chairman, Department Graduate Committee

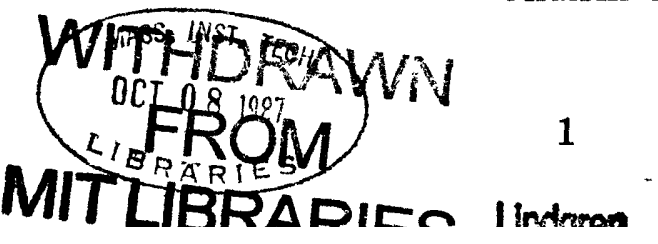




\title{
TIDAL VELOCITY ASYMMETRIES AND BEDLOAD TRANSPORT IN SHALLOW EMBAYMENTS
}

by

\author{
Virginia A. Fry
}

\author{
Submitted to the Department of Earth, \\ Atmospheric, and Planetary Sciences \\ on August 7, 1987 in partial fulfillment of the \\ requirements for the Degree of Master of Science in \\ Oceanography
}

\begin{abstract}
Tidally forced circulation can cause a net near-bed transport of sediment when the tidal velocity is asymmetric about a zero mean (flood or ebb dominant) and the transport rate is nonlinearly related to velocity. The relationship between elevation and velocity is elucidated here to enable one to determine from tide gauge data and sediment transport relations whether tidal asymmetry may cause net sediment transport. Tidal elevation and tidal velocity are related through the equations of motion of the fluid. If the estuary is shallow, the change in cross-sectional area of the channel with the tide is significant with respect to total area: the equations become nonlinear and an exact solution does not exist. A relationship between elevation and velocity in a nonlinear system is derived through the continuity equation and shown to be significantly different than the linear relation. Finite difference numerical solutions of the one dimensional, shallow water nonlinear equations are compared to the continuity relation and are in good agreement.

The relationship between elevation asymmetry and ratio of flood-to-ebb bedload transport is calculated for both the linear relation between elevation and velocity and the nonlinear relation. Results show that the ratio of flood-to-ebb bedload transport as calculated from the nonlinear relation between elevation and velocity is similar to the flood-to-ebb ratio calculated from the linear relation.
\end{abstract}

Thesis Supervisor: Dr. David G. Aubrey

Title: Associate Scientist

Woods Hole Oceanographic Institution 


\section{Acknowledgements}

My time spent as a graduate student at the Woods Hole Oceanographic Institution includes some of my most illuminating experiences. From being one of the first Americans on a military island in China to final exams of 18.444 in the large auditorium at MIT where they essentially shoot off a gun at the beginning and end of the race (oops, exam), my eyes have only become larger. All of these experiences, whether good, bad or different, I hopefully will continue to learn from and appreciate. I thank all of my friends and acquaintances that have made this time worthwhile. Dave Aubrey, whose time, energy, generosity and "ribbing" were always there whenever I could find him, I thank for his support and guidance. Keith

Stolzenbach has played a critical role in providing some realism to my ideas. ("You want to solve all the problems of the world and do it in CHINA too?!*”) Thanks to my family for putting up with too many "Don't know when I'll be home next depends on how my work goes." Sometimes it takes a while to get your priorities straight. And Doug, who has put up with more than his share of my "grumpa", I would like to thank for his ability to get rid of those balls and chains that stop one from realizing what the point is of all of this. 
Contents

1 INTRODUCTION 18

2 SHALLOW WATER EQUATIONS 13

2.1 Linear equations $\ldots \ldots \ldots \ldots \ldots \ldots \ldots \ldots \ldots$

2.2 Nonlinear Equations $\ldots \ldots \ldots \ldots \ldots \ldots \ldots \ldots$

2.2 .1 Continuity Relation $\ldots \ldots \ldots \ldots \ldots \ldots \ldots$

2.2.2 Numerical Method . . . . . . . . . . . . . . 29

2.2.3 Continuity versus Numerical Solutions . . . . . . . 31

3 SEDIMENT TRANSPORT 38

4 ERROR DETERMINATION 46

5 CONCLUSIONS $\quad 49$ 
1 Example of tidal velocity asymmetry as expressed by an amplitude ratio and phase difference and its relation to net bedload transport. 10

2 Channel geometry. . . . . . . . . . . . . . . 15

3 Comparisons between tidal elevation and velocity amplitude ratio and phase difference for a linear system with no friction. . . . . . 18

4 Comparisons between tidal elevation and velocity amplitude ratio and phase difference for a linear system with friction. . . . . . . 20

5 Comparison of the tidal velocity phase differences for the numerical solution, the nonlinear continuity solution and the linear solution. .

6 Comparisons of the tidal velocity amplitude ratio for the numerical solution, the nonlinear continuity solution and the linear solution. .

7 Comparisons of the tidal velocity fundamental and harmonic amplitudes of the numerical solutions, the nonlinear continuity solution and the linear solution. . . . . . . . . . . . . . 34

8 Numerical solution for the elevation amplitude of the fundamental and harmonic frequencies. . . . . . . . . . . . . 36

9 Comparisons of the amplitude ratio and phase difference between the numerical solution, the nonlinear continuity solution and the linear solution for a channel with a flat bottom and sloping side walls. . . .

10 Tidal elevation asymmetry versus flood-to-ebb ratio of bedload sediment transport in a linear system with a critical velocity equal to zero. . . . . . . . . . . . . . . . . . . . .

11 Tidal elevation asymmetry versus flood-to-ebb bedload sediment transport in a nonlinear system with a critical velocity equal to zero. . . . 42 
12 An illustration of how the difference between the nonlinear and the linear solutions for the velocity amplitude ratio and the phase difference cancel each other in the calculation of the flood-to-ebb bedload transport ratio. ...................... 4

13 Tidal elevation asymmetry versus flood-to-ebb ratio of bedload sediment transport in a linear system with a critical velocity equal to 20 $\mathrm{cm} / \mathrm{sec} \ldots \ldots \ldots \ldots \ldots \ldots \ldots \ldots$

14 Tidal elevation asymmetry versus flood-to-ebb ratio of bedload sediment transport in a nonlinear system with a critical velocity equal to $20 \mathrm{~cm} / \mathrm{sec} . \ldots \ldots \ldots \ldots \ldots$

15 Error determination of the nonlinear continuity solution in terms of the ratio of flood-to-ebb bedload transport. . . . . . . . . . . 48 
1 Differences between the linear and nonlinear relations between elevation and velocity, and their effect on the flood-to-ebb bedload transport ratio. ....................... 44 


\section{INTRODUCTION}

Embayments comprise a large fraction of the coastline and historically have been areas of intensive use, resulting in degraded environmental quality and substantial reduction in living marine resources (Cloern and Nichols, 1985). Appropriate and effective management of coastal embayments requires research to understand these complex systems.

Circulation processes influence many aspects of an embayment. Sediment and pollutant transport as well as location and type of fisheries all are affected by water circulation. Three dominant types of circulation found in an estuary are thermohaline, wind-driven and tidal. The relative importance of the different types of circulation in transporting sediments or pollutants will vary depending on the particular estuary and the time of year.

Tidal circulation can cause a net transport of sediment if the tidal residual is nonzero or if the tidal velocity is asymmetric about the mean. Tidal currents flowing by topographic features generate residual currents that are expressed by the advective terms in the momentum equations (Tee, 1976; Zimmerman, 1981). A tidal velocity that is asymmetric about a zero mean will be flood or ebb dominant. Flood (ebb) dominance occurs when currents in the flood (ebb) direction are stronger but last for a shorter duration than ebb (flood) currents. A tidal velocity that is flood or ebb dominant may cause a net sediment transport if the transport rate is nonlinearly related to velocity. Aubrey(1986) has calculated the ratio of flood-to-ebb bedload transport as a function of the degree of flood or ebb dominance. The effect of a tidal velocity asymmetry around a zero mean may cause a significant transport asymmetry.

The tidal velocity can be represented by a summation of harmonic functions. 
If the velocity is represented as a combination of a fundamental frequency and an even harmonic, net transport can occur. Any other combination of frequencies will not cause a net transport of sediment over long time scales. For a limited interval of time, frequency combinations other than a fundamental and an even harmonic may cause a net transport but over the period of the beat frequency, net transport will be zero. The degree of asymmetry can be described by an amplitude ratio of the even harmonic to the fundamental and the phase difference between the fundamental and even harmonic. Defining the fundamental and the first even harmonic frequency constituents (sea surface or velocity) as

$$
\begin{gathered}
\text { Fund }=A_{1} \cos \left(\omega t-\theta_{1}\right) \\
\text { Harm }=A_{2} \cos \left(2 \omega t-\theta_{2}\right)
\end{gathered}
$$

the amplitude ratio and the phase difference can be written:

$$
\begin{aligned}
& \frac{A m p_{\text {Harm }}}{A m p_{\text {Fund }}}=\frac{A_{2}}{A_{1}} \\
& \triangle \theta=\theta_{2}-2 \theta_{1} .
\end{aligned}
$$

Figure 1 gives examples of how the amplitude ratio and phase difference expresses a velocity asymmetry and how a velocity asymmetry causes a net bedload transport. Flood dominance occurs when $\Delta \theta_{\text {vel }}$ is between $270^{\circ}$ and $90^{\circ}$ with maximum asymmetry at $0^{\circ}$; ebb dominance occurs when $\Delta \theta_{v e l}$ is between $90^{\circ}$ and $270^{\circ}$ with maximum asymmetry at $180^{\circ}$. The closer the amplitude ratio is to 1 , the lesser the asymmetry.

In a linear system, an elevation asymmetry and thus a velocity asymmetry occur only when the system is being forced by both a fundamental and an even harmonic frequency. A linear system can change the amplitude and phase of a frequency component but cannot produce frequencies that are not already in the system. In a 

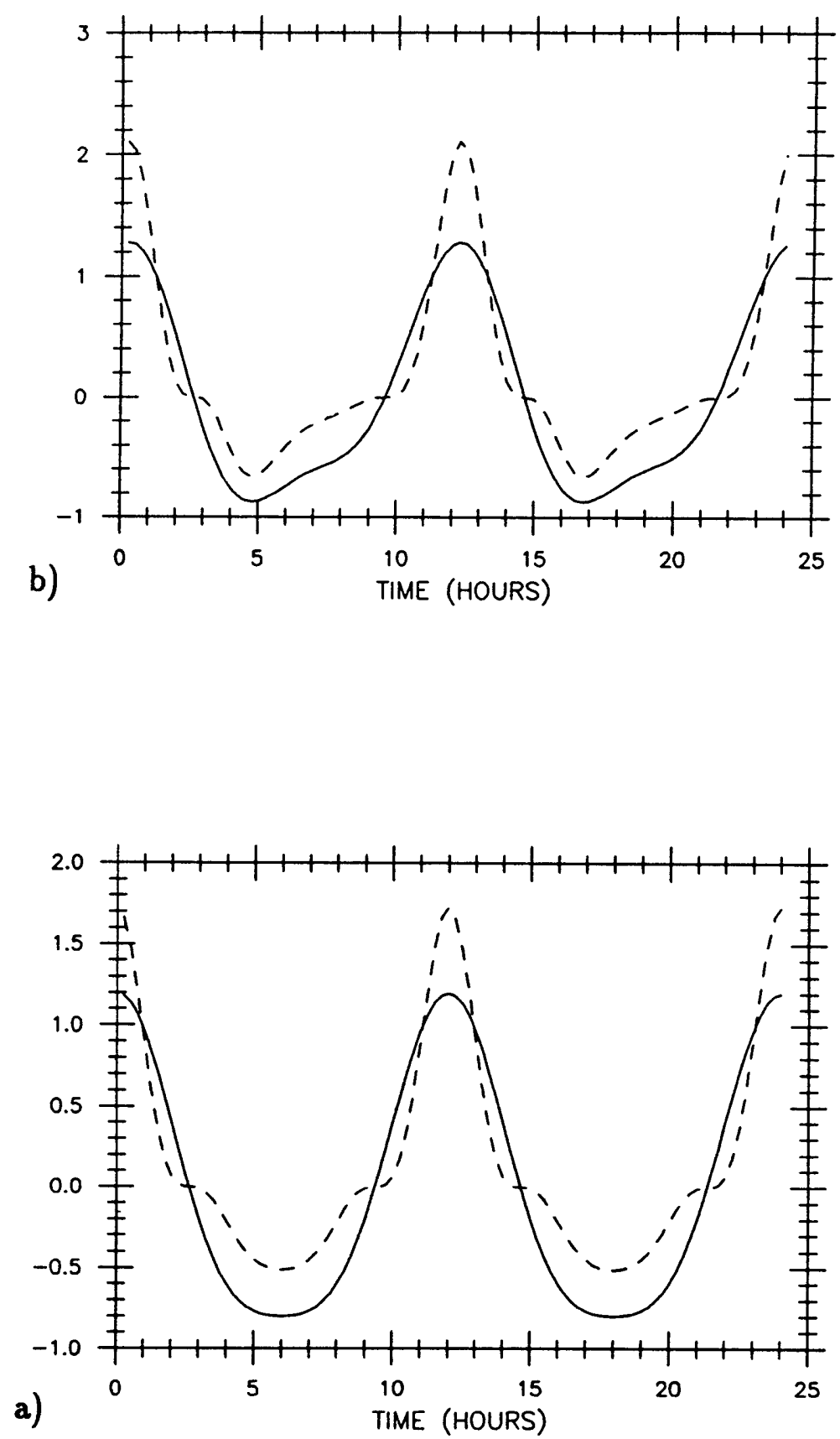

Figure 1: Example of tidal velocity asymmetry as expressed by an amplitude ratio and phase difference and its relation to net bedload transport. (Velocity is solid line and the bedload transport relation to velocity $\left(v^{3}\right)$ is dashed line. The flood-to-ebb ratio is not dependent on the velocity units therefore they are not included in figure.)

a) $\left.\frac{A m p_{H, \text { vel }}}{A m p_{F, \text { oll }}}=.2, \Delta \theta_{\text {vel }}=0^{\circ}, \frac{F \text { lood }}{E b b}=1.97 \mathrm{~b}\right) \frac{A m p_{H, \text { val }}}{A m p_{F, \text { oll }}}=.3, \Delta \theta_{\text {vel }}=-45^{\circ}, \frac{F \text { lood }}{E b b}=2.24$ 
nonlinear system, harmonic frequencies are produced by the fundamental frequency, thus a velocity asymmetry can occur when the system is being forced by only one frequency.

Tidal asymmetries or distortions have been studied previously by analyzing the shallow water nonlinear equations. The nonlinear equations generally do not have an exact solution thus they must be solved using another method. Numerical modeling methods are commonly used to solve nonlinear equations. These methods are not the best for determining the relationship between various parameters of the system because each computation gives just one solution and does not tell what would happen if the parameters were changed. The numerical models must be run many times to determine how the various parameters of the system affect the solution. A perturbation method is another means for solving nonlinear equations, and was used by Kreiss (1957), Gallagher and Munk (1971), and Kabbaj and Le Provost (1980) along with numerical methods to solve the tidally forced shallow water equations. Kreiss (1957) determined that advective and friction terms produce harmonics that cause a tidal distortion. Gallagher and Munk (1971) studied the effects of tidal amplitude, channel geometry and friction on nonlinear distortions in a quasi-linearized set of shallow water equations. Kabbaj and Le Provost (1980) analyzed the importance of quadratic bottom friction in nonlinear tidal distortions. All of these investigations used initial equations that drop nonlinear terms of $O$ (tidal amplitude/water depth) which are significant in our calculations. Speer and Aubrey (1985) and Friedrichs and Aubrey (submitted) related channel geometry to elevation asymmetry through numerical solutions.

The emphasis of this paper is to understand tidal velocity asymmetry, its relation to tidal elevation asymmetry, and the significance of the elevation asymmetry in terms of sediment transport. Theoretically, it is of interest to show the phys- 
ical basis for the nonlinear relation between elevation and velocity and how it is represented in the equations of motion. The height and width of a channel in a shallow water system change significantly with the tide causing a nonlinear relation in the continuity equation. On the more practical side, a relation between elevation and velocity enables one to determine from tide gauge data and sediment transport relations whether tidal asymmetries around a zero mean cause a net transport of sediment. If the tidal asymmetries are significant, an average of the tidal velocity will not be representative of transport processes that are related nonlinearly to velocity. A tidal residual alone should be used to determine the net transport only when the sediment has a transport relation that is linearly related to velocity.

Tidal velocity is related to tidal elevation through the shallow water equations. The 1-dimensional equations, when linear, have analytical solutions that relate velocity to elevation. A relationship between elevation and velocity in a nonlinear system is derived through the continuity equation. A nonlinear relation is calculated between elevation and velocity at the landward end of the channel and the variation in velocity along the channel is represented by the linear solution. The method of using the elevation at the landward end of the channel to determine the velocity is based on the observations from the linear solutions that the elevation asymmetry at the landward end of the channel determines the approximate velocity asymmetry everywhere in the channel. Numerical solutions are calculated to check the validity of this method. Results show that the nonlinear relation between velocity and elevation is significantly different from the linear relation.

In order to determine the significance of tidal asymmetry in transporting sediment, the ratio of flood-to-ebb bedload transport is plotted versus elevation asymmetry data. Suspended load transport due to tidal asymmetries cannot be calculated without defining the size of the sediment and thus is best to calculate 
separately for the particular system of interest.

\section{SHALLOW WATER EQUATIONS}

The equations that define fluid motions in an embayment are conservation of mass and conservation of momentum (Pritchard, 1971). The one dimensional equations are used, not to describe a real system in total but to give insight into one aspect of the problem. Integration of the shallow water equations over depth and width yields

Conservation of mass-

$$
\frac{\partial A}{\partial t}+\frac{\partial U}{\partial x}=0
$$

Conservation of momentum-

$$
\frac{\partial U}{\partial t}=-g A \frac{\partial \eta}{\partial x}-\text { friction term - advective term }
$$

where

$A(x, t)$ is the area of the channel

$U(x, t)$ is the volume flux averaged over depth and width of channel

$\eta(x, t)$ is the elevation of free surface above mean sea level

$g$ is gravitational acceleration

$t$ is time

$x$ is positive upchannel.

These equations define motion in a channel having width, $b$, mean depth, $h$ and length, $L$. The width, $b$, and area, $A$, as shown in Figure 2 are defined as

$$
\begin{gathered}
b=b_{0}+\beta(h+\eta) \\
A=b(h+\eta)=b_{0}(h+\eta)+\beta(h+\eta)^{2}
\end{gathered}
$$


where

$\beta$ is the slope of the side walls (width/height)

$b_{0}$ is the width of the channel bottom.

The channel that is being studied here is open at the ocean end $(x=0)$ and closed at the landward end $(x=L)$. For tidally forced circulation in an embayment, the boundary conditions are

$$
\begin{gathered}
U=0 \text { at } x=L \\
\eta=\eta_{1} \cos (k L) \cos (\omega t)+\eta_{2} \cos (2 k L) \cos (2 \omega t-\theta) \text { at } x=0
\end{gathered}
$$

where

$\eta_{1}, \eta_{2}$ are the amplitude of the fundamental and first harmonic frequency of elevation at the landward end of the channel $(x=L)$

$\omega$ is the fundamental frequency

$k$ is the wave number $(k=\omega / c, c=\sqrt{g h})$

$\theta$ is the negative of the phase difference between the fundamental and harmonic frequency at the ocean end of the channel.

\subsection{Linear equations}

In a linear system, an elevation asymmetry and thus a velocity asymmetry occur only when the system is being forced by both a fundamental and an even harmonic frequency. The equations are linear if width, $b$, and area, $A$, are constant in space and time, a linear friction term is used and the nonlinear advective terms are neglected. If $\eta / h \ll 1$ then width, $b$, and area, $A$, are approximately constant. The linearized shallow water equations are

$$
\frac{\partial \eta}{\partial t}+\frac{1}{b_{0}} \frac{\partial U}{\partial x}=0
$$



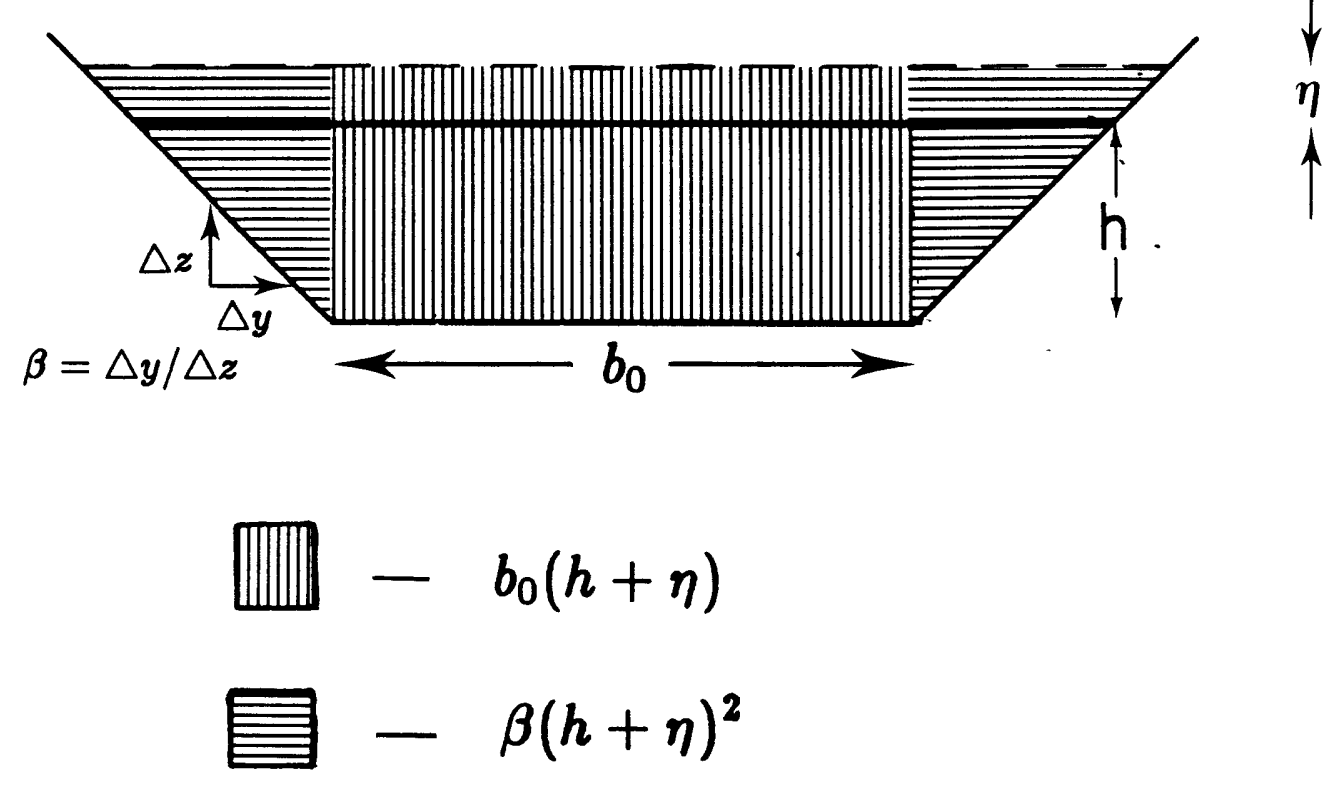

Figure 2: Channel geometry. The area of the channel that is marked by the vertical stippling is the area that changes in height due to a change in tidal elevation: the horizontal stippling indicates the area of the channel that changes in height and width due to a change in tidal elevation. 


$$
\frac{\partial U}{\partial t}=-g A_{0} \frac{\partial \eta}{\partial x}-f U
$$

where

$f=$ linear friction factor $\left(t^{-1}\right)$.

The 1-dimensional linear equations of motion can be combined into the wave equation when the friction term is neglected.

$$
\frac{\partial^{2} \eta}{\partial t^{2}}=\left(\frac{\omega}{k}\right)^{2} \frac{\partial^{2} \eta}{\partial x^{2}}
$$

The solution to the wave equation with the above boundary conditions (Equations 4 and 5) is a standing wave where

$$
\begin{gathered}
\eta=\eta_{1} \cos (k(x-L)) \cos (\omega t)+\eta_{2} \cos (2 k(x-L)) \cos (2 \omega t-\theta) \\
U=b c \eta_{1} \sin (k(x-L)) \sin (\omega t)+b c \eta_{2} \sin (2 k(x-L)) \sin (2 \omega t-\theta)
\end{gathered}
$$

The elevation asymmetry in a linear system having no friction is represented by the amplitude ratio and the phase relationship. The amplitude ratio of the first harmonic $\left(H_{1}\right)$ to the fundamental frequency $(F)$ is

$$
\frac{A m p_{H_{1}, \text { elev }}}{A m p_{F, \text { elev }}}=\frac{\eta_{2}}{\eta_{1}} \frac{\cos (2 k(x-L))}{\cos (k(x-L))} \approx \frac{\eta_{2}}{\eta_{1}}
$$

For channel lengths of $O(10 \mathrm{~km})$ and water depth of $\mathrm{O}(3 \mathrm{~m}), k(x-L)$ is small and thus $\cos (k(x-L))$ is approximately one. The phase relationship between the fundamental and the first harmonic is

$$
\Delta \theta_{\text {elev }}=\theta_{H_{1}, \text { elev }}-2 \theta_{F, \text { elev }}=\theta
$$

where

$\theta_{F, e l e v}$ is the phase of the fundamental frequency in elevation, set to 0 in standing wave equation for ease of calculations, $\theta_{H_{1} \text {,elev }}$ is the phase of the first harmonic frequency in elevation, equal to $\theta$ in standing 
wave equation.

The velocity asymmetry due to a linear system having no friction is represented by the amplitude ratio and the phase difference. The amplitude ratio of the first harmonic to the fundamental is

$$
\frac{A m p_{H_{1}, \text { el }}}{A m p_{F, \text { vel }}}=\frac{\eta_{2}}{\eta_{1}} \frac{\sin (2 k(x-L))}{\sin (k(x-L))} \approx 2 \frac{\eta_{2}}{\eta_{1}}
$$

Since $k(x-L)$ is small, $\sin x \approx x$. The phase relationship between the fundamental and the first harmonic is

$$
\Delta \theta_{\text {vel }}=\theta_{H_{1}, \text { vel }}-2 \theta_{F, \text { vel }}+90^{\circ}=\theta+90^{\circ} \text {, }
$$

where

$\theta_{F, v e l}$ is the phase of the fundamental frequency in velocity, equal to zero in the standing wave equation

$\theta_{H_{1}, \text { vel }}$ is the phase of the first harmonic frequency in velocity, equal to $\theta$ in the standing wave equation.

The calculation of $\Delta \theta_{\text {vel }}$ is shifted by $90^{\circ}$ from $\Delta \theta_{\text {elev }}$ due to velocity being represented by sine functions. The amplitude ratio and phase relationship for both elevation and velocity of a linear solution with no friction are depicted in Figure 3.

The solution to the linear equations (Equations 6 and 7) with the friction term not equal to zero has been solved by Dronkers(1964). Dronkers' solution gives a sea surface elevation,

$$
\eta=\begin{aligned}
\eta_{1} \frac{C_{F} \cos \omega t+D_{F} \sin \omega t}{\cosh \left(s \delta_{F}\right)+\cos \left(s \epsilon_{F}\right)}+ \\
\eta_{2} \frac{C_{H} \cos 2 \omega t+D_{H} \sin 2 \omega t}{\cosh \left(s \delta_{H}\right)+\cos \left(s \epsilon_{H}\right)}
\end{aligned}
$$



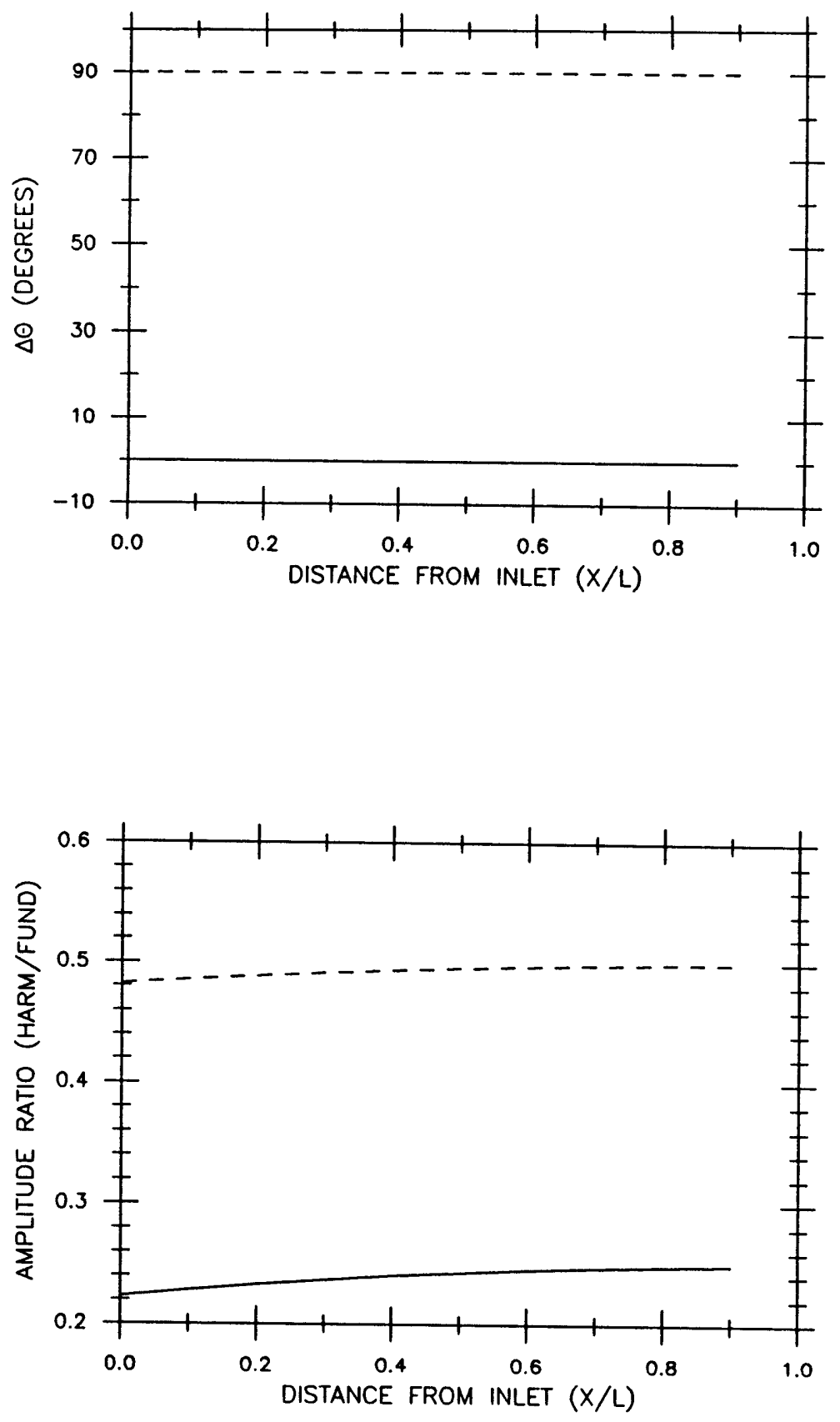

Figure 3: Comparisons between tidal elevation (-) and velocity (- - -) amplitude ratio and phase difference for a linear system with no friction. $\left(A m p_{F \text { und,inlet, elev }}=0.8 \cos (k L), A m p_{H \text { arm, inlet, alev }}=0.2 \cos (2 k L), \Delta \theta_{\text {inlet, elev }}=0^{\circ}\right.$, $h=3.0$ meters, $L=10 \mathrm{~km}$., $b_{0}=50$ meters, $\beta=0$ ) 
and a volume flux,

$$
\begin{aligned}
U= & \eta_{1} \frac{b_{0} c_{0}}{\delta_{F}^{2}+\epsilon_{F}^{2}} \frac{E_{F} \cos \omega t+F_{F} \sin \omega t}{\cosh \left(s \delta_{F}\right)+\cos \left(s \epsilon_{F}\right)}+ \\
& \eta_{2} \frac{b_{0} c_{0}}{\delta_{H}^{2}+\epsilon_{H}^{2}} \frac{C_{H} \cos 2 \omega t+D_{H} \sin 2 \omega t}{\cosh \left(s \delta_{H}\right)+\cos \left(s \epsilon_{H}\right)}
\end{aligned}
$$

in which

$$
\begin{aligned}
C_{i}= & 2\left[\varphi_{1, i}(L) \varphi_{1, i}(x-L)+\varphi_{2, i}(L) \varphi_{2, i}(x-L)\right] \\
D_{i}= & -2\left[\varphi_{1, i}(L) \varphi_{2, i}(x-L)-\varphi_{2, i}(L) \varphi_{1, i}(x-L)\right] \\
E_{i}= & 2 \delta\left[\varphi_{1, i}(L) \varphi_{3, i}(x-L)-\varphi_{2, i}(L) \varphi_{4, i}(x-L)\right] \\
& -2 \epsilon\left[\varphi_{1, i}(L) \varphi_{4, i}(x-L)+\varphi_{2, i}(L) \varphi_{3, i}(x-L)\right] \\
F_{i}= & 2 \epsilon\left[\varphi_{1, i}(L) \varphi_{3, i}(x-L)-\varphi_{2, i}(L) \varphi_{1, i}(x-L)\right] \\
& +2 \delta_{i}\left[\varphi_{1, i}(L) \varphi_{1, i}(x-L)+\varphi_{2, i}(L) \varphi_{3, i}(x-L)\right] \\
& i=F(\text { fundamental) or H(harmonic) }
\end{aligned}
$$

and where

$$
\begin{gathered}
\varphi_{1, i}(x)=\cosh \left(\frac{1}{2} s \delta_{i} \frac{x}{L}\right) \cos \left(\frac{1}{2} s \epsilon_{i} \frac{x}{L}\right) \\
\varphi_{2, i}(x)=\sinh \left(\frac{1}{2} s \delta_{i} \frac{x}{L}\right) \sin \left(\frac{1}{2} s \epsilon_{i} \frac{x}{L}\right) \\
\varphi_{3, i}(x)=\cosh \left(\frac{1}{2} s \delta_{i} \frac{x}{L}\right) \sin \left(\frac{1}{2} s \epsilon_{i} \frac{x}{L}\right) \\
\varphi_{4, i}(x)=\sinh \left(\frac{1}{2} s \delta_{i} \frac{x}{L}\right) \cos \left(\frac{1}{2} s \epsilon_{i} \frac{x}{L}\right) \\
\delta_{i}=\left[-0.5+0.5\left(1+\left(\frac{f}{\omega_{i}}\right)^{2}\right)^{1 / 2}\right]^{1 / 2} \\
\epsilon_{i}=\left[0.5+0.5\left(1+\left(\frac{f}{\omega_{i}}\right)^{2}\right)^{1 / 2}\right]^{1 / 2} \\
s=\frac{4 \pi L}{\lambda_{\text {tide }}}
\end{gathered}
$$



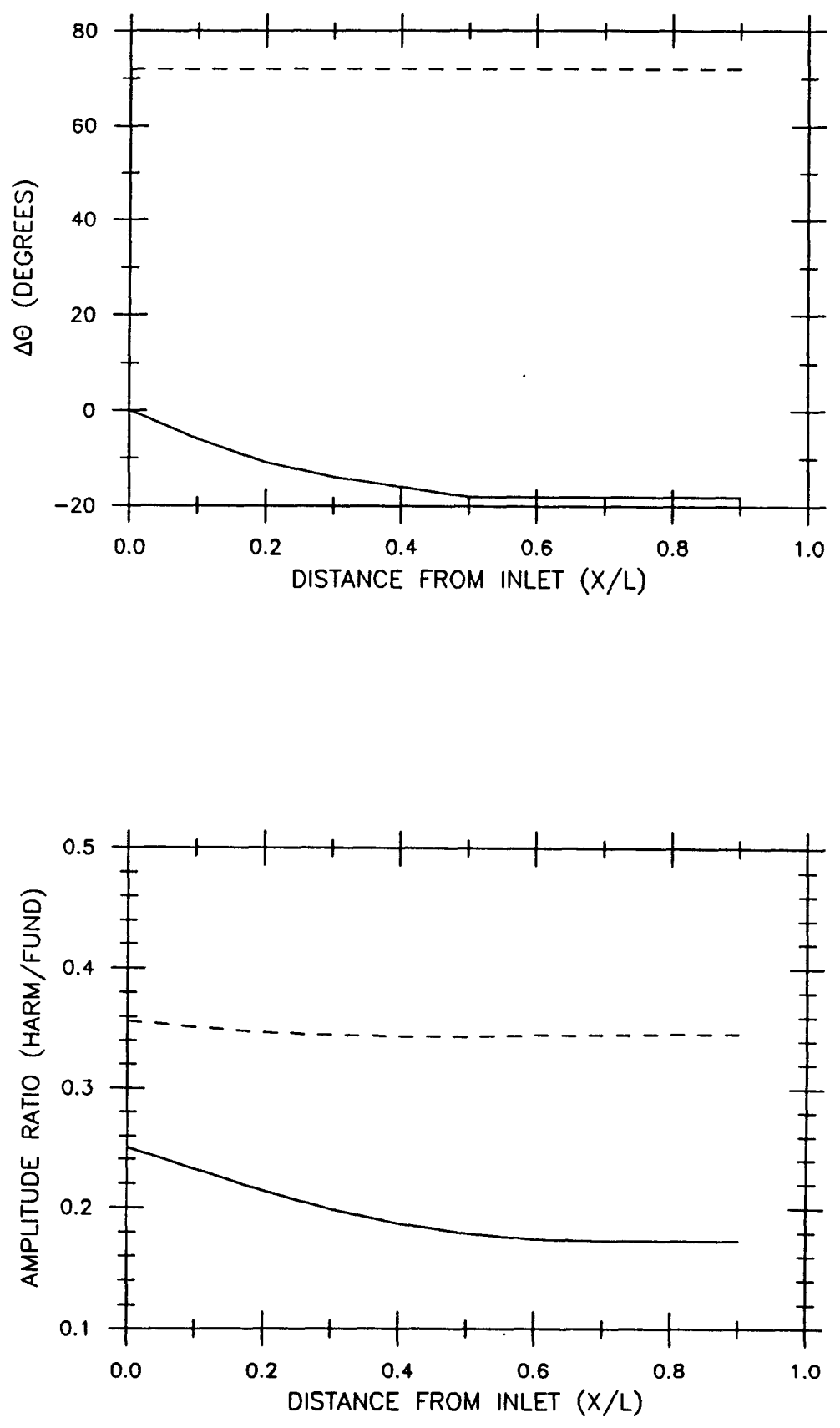

Figure 4: Comparisons between tidal elevation (-) and velocity (- - ) amplitude ratio and phase difference for a linear system with friction. $\left(A m p_{F}\right.$ und,inlet, elev $=0.8$, $A m p_{\text {Harm,inlet,elev }}=0.2, \Delta \theta_{\text {inlet,elev }}=0^{\circ}, h=3.0$ meters, $L=10 \mathrm{~km}$., $b_{0}=50$ meters, $\beta=0$ ) 
Results of Dronker's solution in terms of velocity asymmetry related to elevation asymmetry are depicted in Figure 4.

A linear system of the shallow water equations with and without friction shows the following velocity asymmetry as compared to elevation asymmetry. The amplitude ratio of the first harmonic to the fundamental is

$$
\frac{A m p_{H_{1}, \text { vel }}}{A m p_{F, \text { vel }}} \approx 2{\frac{A m p_{H_{1}, \text { elev }}}{A m p_{F, \text { elev }}(x=L)}}
$$

The phase relationship between the fundamental and the first harmonic is

$$
\Delta \theta_{\text {vel }}=\Delta \theta_{\text {elev },(x=L)}+90^{\circ}
$$

Linear solutions show that the relationship between elevation asymmetry and velocity asymmetry (Equations 8 and 9 ) holds best toward the landward end of the channel. In linear solutions, velocity asymmetry varies insignificantly down the channel for a channel geometry on the order of 10 kilometers in length and 3 meters in depth (Figures 3 and 4). Linear solutions thus show that velocity asymmetry everywhere in the channel can be determined by the elevation asymmetry at the landward end of the channel.

\subsection{Nonlinear Equations}

The shallow water equations (Equations 1 and 2) are nonlinear when area, $A$, or width, $b$, are not constant in time or space, or when the friction term and/or advection term is nonlinear. The velocity asymmetry is determined from the elevation asymmetry in the nonlinear equations by using an elevation that is representative of the elevation at the landward end of the channel when $\mathrm{x}=\mathrm{L}$ and computing the velocity through the continuity equation. This method is based on the observations from the linear solutions that the elevation asymmetry at the landward end of the 
channel determines the velocity asymmetry everywhere in the channel. Although the asymmetry in a linear system is produced by a tidal forcing of a fundamental and an even harmonic frequency at the inlet, and the asymmetry in a nonlinear system can be due to the forcing of only a fundamental frequency at the inlet and generation of the harmonic frequency within the channel the validity of this method will be tried and checked by comparisons with numerical solutions.

\subsubsection{Continuity Relation}

Tidal velocity is determined from a tidal elevation that is representative of the elevation at the landward end of the channel $(x=L)$. This elevation is expressed as

$$
\begin{aligned}
\eta= & \eta_{1} \cos (k(x-L)) \cos (\omega t)+\eta_{2} \cos (2 k(x-L)) \cos (2 \omega t-\theta)+ \\
& \eta_{3} \cos (3 k(x-L)) \cos (3 \omega t-\zeta)
\end{aligned}
$$

The second harmonic $(3 \omega t)$ is included in this expression because it is possible to create a first harmonic $(2 \omega t)$ from nonlinear terms that multiply the fundamental $(\omega t)$ and the second harmonic $(3 \omega t)$. The velocity due to this elevation asymmetry is calculated from the volume flux which is found using the continuity equation. VERTICAL SIDE WALLS

A channel with vertical side walls has an area that is expressed as

$$
A=b_{0}(h+\eta)
$$

where $b_{0}$ is the width of the channel. The volume flux is calculated through the continuity equation from the tidal elevation (Eqn. 10);

$$
\frac{\partial U}{\partial x}=-\frac{\partial A}{\partial t} \quad \text { (continuity equation) }
$$




$$
\begin{aligned}
& \frac{\partial A}{\partial t}=b_{0} \frac{\partial \eta}{\partial t} \\
& \frac{\partial A}{\partial t}=-b_{0} h\left[\left(\omega \eta_{1} / h\right) \cos (k(x-L)) \sin (\omega t)+\left(2 \omega \eta_{2} / h\right) \cos (2 k(x-L)) \sin (2 \omega t-\theta)+\right. \\
& \left.\left(3 \omega \eta_{3} / h\right) \cos (3 k(x-L)) \sin (3 \omega t-\varsigma)\right] \\
& U=\int-\frac{\partial A}{\partial t} d x \\
& U=b_{0} h(\omega / k)\left[\left(\eta_{1} / h\right) \sin (k(x-L)) \sin (\omega t)+\left(\eta_{2} / h\right) \sin (2 k(x-L)) \sin (2 \omega t-\theta)+\right. \\
& \left.\left(\eta_{3} / h\right) \sin (3 k(x-L)) \sin (3 \omega t-\zeta)\right]+C
\end{aligned}
$$

Since $U=0$ at $x=L$, then $C=0$.

Velocity asymmetry can be determined from volume flux by

$$
\text { Velocity }=V=U / A,
$$

with a binomial expansion,

$$
\frac{1}{A}=\frac{1}{b_{0} h}\left(1-\frac{\eta}{h}+O\left(\frac{\eta_{1}}{h}\right)^{2}\right)
$$

$$
\begin{aligned}
V= & \frac{\omega}{k} \frac{\eta_{1}}{h}\left[\sin (k(x-L)) \sin (\omega t)+\left(\eta_{2} / \eta_{1}\right) \sin (2 k(x-L)) \sin (2 \omega t-\theta)-\right. \\
& \left.\left(\eta_{1} / 4 h\right) \sin (2 k(x-L)) \sin (2 \omega t)+O\left(\left(\eta_{1} / h\right)^{2}\right)+O\left(\eta_{2} / h\right)\right]
\end{aligned}
$$

Two sinusoidal functions having the same frequency but different amplitudes and phases can be expressed as a single function of the same frequency with one amplitude and phase.

$$
\begin{aligned}
F \sin (2 \omega t)+G \sin (2 \omega t-\theta) & =(F+G \cos \theta) \sin (2 \omega t)-(G \sin \theta) \cos (2 \omega t) \\
& =\left[(F+G \cos \theta)^{2}+(G \sin \theta)^{2}\right]^{1 / 2} \sin (2 \omega t-\phi)
\end{aligned}
$$




$$
\phi=\arctan \left(\frac{F+G \cos \theta}{-G \sin \theta}\right)-90^{\circ}
$$

In tidal velocity

$$
\begin{gathered}
F=-\frac{\eta_{1}}{4 h} \sin (2 k(x-L)), \\
G=\frac{\eta_{2}}{\eta_{1}} \sin (2 k(x-L)),
\end{gathered}
$$

thus

$$
\begin{aligned}
V= & \frac{\omega}{k} \frac{\eta_{1}}{h}\{\sin (k(x-L)) \sin (\omega t)+ \\
& {\left[\left(\frac{\eta_{2}}{\eta_{1}} \cos \theta-\frac{\eta_{1}}{4 h}\right)^{2}+\left(\frac{\eta_{2}}{\eta_{1}} \sin \theta\right)^{2}\right]^{1 / 2} \sin (2 k(x-L)) \sin (2 \omega t-\phi)+} \\
& \left.O\left(\left(\eta_{1} / h\right)^{2}\right)+O\left(\eta_{2} / h\right)\right\}
\end{aligned}
$$

where

$$
\phi=\arctan \left(\frac{\frac{\eta_{2}}{\eta_{1}} \cos \theta-\frac{\eta_{1}}{4 h}}{-\frac{\eta_{2}}{\eta_{1}} \sin \theta}\right)-90^{\circ} .
$$

The velocity asymmetry for a nonlinear system with a channel having vertical side walls is represented by the following equations. The amplitude ratio of the first harmonic to the fundamental is

$$
\frac{A m p_{H_{1}, \text { el }}}{A m p_{F, \text { el }}}=\frac{\sin (2 k(x-L))}{\sin (k(x-L))}\left[\left(\frac{\eta_{2}}{\eta_{1}} \cos \theta-\frac{\eta_{1}}{4 h}\right)^{2}+\left(\frac{\eta_{2}}{\eta_{1}} \sin \theta\right)^{2}\right]^{1 / 2} .
$$

The phase relationship between the fundamental and the first harmonic in the velocity equation when velocity is expressed as a summation of sine functions is

$$
\Delta \theta_{\text {vel }}=\theta_{H_{1}}-2 \theta_{F, v e l}+90^{\circ}
$$

The phase of the fundamental frequency in the velocity equation is zero $\left(\theta_{F, v e l}=0\right)$ and the phase of the harmonic frequency is $\phi\left(\theta_{H_{1}, v a l}=\phi\right)$, thus

$$
\Delta \theta_{\text {vel }}=\arctan \left(\frac{\frac{\eta_{2}}{\eta_{1}} \cos \theta-\frac{\eta_{1}}{1 h}}{-\frac{\eta_{2}}{\eta_{1}} \sin \theta}\right)
$$


But

$$
\begin{gathered}
\tan \left(180^{\circ}+A\right)=\tan (A), \\
\arctan ()=180^{\circ}+A \text { or } A,
\end{gathered}
$$

therefore

$$
\Delta \theta_{\text {vel }}=\arctan \left(\frac{\frac{\eta_{2}}{\eta_{1}} \cos \theta-\frac{\eta_{1}}{4 h}}{-\frac{\eta_{2}}{\eta_{1}} \sin \theta}\right)+0^{\circ} \text { or } 180^{\circ} .
$$

Choose $0^{\circ}$ or $180^{\circ}$ so $\Delta \theta_{\text {vel }} \approx \Delta \theta_{\text {elev }}+90^{\circ}$ The velocity asymmetry should be calculated with tidal elevation parameters from the landward end of the channel. The tidal elevation parameters are the following:

$\eta_{1}$ is the tidal elevation amplitude of the fundamental

frequency at $\mathrm{x}=\mathrm{L}$

$\eta_{2}$ is the tidal elevation amplitude of the first

harmonic frequency at $\mathrm{x}=\mathrm{L}$

$\theta$ is the phase difference between the fundamental and harmonic

frequencies of the tidal elevation at $x=L$

If the tidal elevation is expressed as a summation of cosine functions (i.e. equation 10),

$$
\Delta \theta_{\text {elev }}=\theta_{H, \text { elev }}-2 \theta_{F, e l e v}
$$

Since in our calculations, $\theta_{F, \text { elev }}$ was set equal to zero for ease of computation and $\theta_{H, \text { elev }}=\theta$,

$$
\theta=\Delta \theta_{\text {elev }}
$$

The $\theta$ to use for the velocity asymmetry calculation is equal to $\Delta \theta_{\text {elev }}$ calculated from tidal elevation data at the landward end of the channel. 


\section{SLOPING SIDE WALLS}

A channel with sloping side walls has an area that is expressed by Equation 3. The volume flux due to the elevation asymmetry (Equation 10) is calculated through the continuity equation:

$$
\begin{gathered}
\frac{\partial U}{\partial x}=-\frac{\partial A}{\partial t} \\
\frac{\partial A}{\partial t}=b_{0} \frac{\partial \eta}{\partial t}+2 \beta(h+\eta) \frac{\partial \eta}{\partial t} \\
U=-\left(b_{0}+2 \beta h\right) \int \frac{\partial \eta}{\partial t} d x-2 \beta \int \eta \frac{\partial \eta}{\partial t} d x \\
\int \frac{\partial \eta}{\partial t} d x=-(\omega / k)\left[\eta_{1} \sin (k(x-L)) \sin (\omega t)+\eta_{2} \sin (2 k(x-L)) \sin (2 \omega t-\theta)+\right. \\
\left.\eta_{3} \sin (3 k(x-L)) \sin (3 \omega t-\zeta)\right]+C_{1} \\
\int \eta \frac{\partial \eta}{\partial t} d x=-(\omega / k)\left(\eta_{1}^{2} / 8\right)[(2 k(x-L)+\sin (2 k(x-L))) \sin (2 \omega t)]+ \\
O\left(\eta_{2} \eta_{1}\right)+C_{2} \\
U=\left(b_{0}+2 \beta h\right) \frac{\omega}{k}\left[\eta_{1} \sin (k(x-L)) \sin (\omega t)+\eta_{2} \sin (2 k(x-L)) \sin (2 \omega t-\theta)\right] \\
+\frac{1}{4} \beta \eta_{1}^{2} \omega / k[2 k(x-L)+\sin (2 k(x-L))] \sin (2 \omega t)+O\left(\eta_{2} \eta_{1}\right)+C_{3}
\end{gathered}
$$

Since $U=0$ at $x=L$, then $C_{3}=0$.

The velocity is determined from the volume flux by

$$
\text { Velocity }=V=U / A \text {, }
$$

where

$$
\frac{1}{A}=\frac{1}{b_{0}(h+\eta)+\beta(h+\eta)^{2}} .
$$

A v-shaped channel $\left(b_{0}=0\right)$ simplifies the expansion of $1 / \mathrm{A}$ and therefore the calculation of the velocity as shown in the following equations:

$$
\begin{gathered}
\frac{1}{A}=\frac{1}{\beta(h+\eta)^{2}} \\
\frac{1}{A}=\frac{1}{\beta h^{2}}\left(1-\frac{2 \eta}{h}+O\left(\left(\frac{\eta_{1}}{h}\right)^{2}\right)\right)
\end{gathered}
$$




$$
\begin{aligned}
U= & 2 \beta h^{2}(\omega / k)\left(\eta_{1} / h\right)\left\{\sin (k(x-L)) \sin (\omega t)+\eta_{2} / \eta_{1} \sin (2 k(x-L)) \sin (2 \omega t-\theta)+\right. \\
& \left.\left(\eta_{1} / 8 h\right)[2 k(x-L)+\sin (2 k(x-L))] \sin (2 \omega t)+O\left(\eta_{2} / h\right)\right\} \\
V= & U / A
\end{aligned}
$$

$$
\begin{aligned}
V= & 2(\omega / k)\left(\eta_{1} / h\right)\{\sin (k(x-L)) \sin (\omega t)+ \\
& \left(\eta_{2} / \eta_{1}\right) \sin (2 k(x-L)) \sin (2 \omega t-\theta)+ \\
& \left(\eta_{1} / 8 h\right)[2 k(x-L)+\sin (2 k(x-L))] \sin (2 \omega t)- \\
& \left(\eta_{1} / 2 h\right) \sin (2 k(x-L)) \sin (2 \omega t)+ \\
& \left.O\left(\eta_{2} / h\right)+O\left(\left(\eta_{1} / h\right)^{2}\right)\right\} \\
V= & 2(\omega / k)\left(\eta_{1} / h\right)\{\sin (k(x-L)) \sin (\omega t)+ \\
& \left(\eta_{2} / \eta_{1}\right) \sin (2 k(x-L)) \sin (2 \omega t-\theta)+ \\
& \left(\eta_{1} / 8 h\right)(2 k(x-L)) \sin (2 \omega t)- \\
& \left(3 \eta_{1} / 8 h\right) \sin (2 k(x-L)) \sin (2 \omega t)+ \\
& \left.O\left(\eta_{2} / h\right)+O\left(\left(\eta_{1} / h\right)^{2}\right)\right\}
\end{aligned}
$$

As shown previously in the section on vertical side walls, two sinusoidal functions having the same frequency but different amplitudes and phases can be expressed as a single function of the same frequency with one amplitude and phase.

$$
\begin{gathered}
F \sin (2 \omega t)+G \sin (2 \omega t-\theta)=(F+G \cos \theta) \sin (2 \omega t)-(G \sin \theta) \cos (2 \omega t) \\
=\left[(F+G \cos \theta)^{2}+(G \sin \theta)^{2}\right]^{1 / 2} \sin (2 \omega t-\phi) \\
\phi=\arctan \left(\frac{F+G \cos \theta}{-G \sin \theta}\right)-90^{\circ} \\
F=\left(\frac{1}{8} \frac{2 k(x-L) \eta_{1}}{\sin (2 k(x-L)) h}-\frac{3 \eta_{1}}{8 h}\right) \sin (2 k(x-L))
\end{gathered}
$$




$$
G=\frac{\eta_{2}}{\eta_{1}} \sin (2 k(x-L))
$$

The velocity asymmetry for a nonlinear system with sloping side walls is represented by the following equations. The amplitude ratio of the first harmonic to the fundamental is

$$
\frac{A m p_{H_{1}, \text { el }}}{A m p_{F, v e l}}=\frac{\sin (2 k(x-L))}{\sin (k(x-L))}\left[\left(\frac{\eta_{2}}{\eta_{1}} \cos \theta-\frac{3 \eta_{1}}{8 h}+\frac{1}{8} \frac{2 k(x-L) \eta_{1}}{\sin (2 k(x-L)) h}\right)^{2}+\left(\frac{\eta_{2}}{\eta_{1}} \sin \theta\right)^{2}\right]^{1 / 2}
$$

The phase relationship between the fundamental and the first harmonic is

$$
\begin{aligned}
\Delta \theta_{\text {vel }}= & \arctan \left(\frac{\frac{\eta_{2}}{\eta_{1}} \cos \theta-\frac{3 \eta_{1}}{8 h}+\frac{2 k(z-L) \eta_{1}}{8 \sin (2 k(z-L)) h}}{-\frac{\eta_{2}}{\eta_{1}} \sin \theta}\right) \\
& +0^{\circ} \text { or } 180^{\circ}
\end{aligned}
$$

Choose $0^{\circ}$ or $180^{\circ}$ so that $\Delta \theta_{\text {vel }}$ leads $\Delta \theta_{\text {elev }}$ in phase by approximately $90^{\circ}$.

The velocity asymmetry determined for a v-shaped channel geometry is similar to the solution for a rectangular channel. The difference between the two solutions exists when $2 k(x-L) \neq \sin (2 k(x-L))$. This difference is small for channel lengths much less than the tidal wavelength which is true for the channels considered here.

Channels with geometries that are not rectangular or v-shaped but somewhere in between (i.e., flat bottom with sloping side walls) are more likely to be found in the field. Determination of the velocity asymmetry for a channel having a flat bottom and sloping sides becomes more difficult mathematically due to the binomial expansion of 1 /Area,

where

$$
\text { Area }=b_{0}(h+\eta)+\beta(h+\eta)^{2} .
$$

A rectangular channel has an area where $\beta$ is zero and a v-shaped channel has an area where $b_{0}$ is zero. Channels with flat bottoms and sloping side walls require both terms to express the area of the channel. Numerical solutions for geometries 
between a rectangular and v-shaped channel will be shown in the section comparing numerical and theoretical results.

\subsubsection{Numerical Method}

The validity of the theoretical solutions will be checked by a comparison with numerical solutions. The nonlinear shallow water equations were solved numerically using a quadratic friction term in the momentum equation.

$$
\text { friction term }=\frac{f|U| U P}{A^{2}}
$$

where

$\mathrm{f}$ is the friction factor

$P$ is the wetted perimeter $\left(P=b_{0}+2\left[\beta(h+\eta)^{2}+(h+\eta)^{2}\right]^{1 / 2}\right)$

A quadratic friction term with a constant friction factor, although commonly used (e.g.,Uncles, 1981; Speer, 1984), has its drawbacks. It is felt that odd harmonics of the fundamental frequency are overemphasized (Prandle, 1980), although Pingree and Maddocks (1978) have reproduced $M_{2}$ and $M_{4}$ amplitudes and phases accurately. The quadratic friction term will be used in the numerical solution to determine the relation between elevation and velocity.

A numerical solution of the nonlinear equations is found via an explicit finite difference model using a forward-time, centered-space scheme (Speer, 1984). Sea surface elevation and volume flux are staggered spatially. The accuracy of this scheme is $O\left(\Delta t, \Delta x^{2}\right)$. The equations in their discrete form are given below:

$$
\begin{gathered}
\frac{\partial A}{\partial t}+\frac{\partial U}{\partial x}=0 \\
A=b_{0}(h+\eta)+\beta(h+\eta)^{2} \\
\frac{\partial A}{\partial t}=\left[b_{0}+2 \beta(h+\eta)\right] \frac{\partial \eta}{\partial t}
\end{gathered}
$$




$$
\begin{gathered}
\frac{\partial \eta}{\partial t}+\frac{1}{b_{0}+2 \beta(h+\eta)} \frac{\partial U}{\partial x}=0 \\
\frac{\eta_{j+1 / 2}^{n+1}-\eta_{j+1 / 2}^{n}}{\Delta t}=-\frac{1}{b_{0}+2 \beta(h+\eta)_{j+1 / 2}^{n}} \frac{U_{j+1}^{n}-U_{j}^{n}}{\Delta x} \\
\frac{\partial U}{\partial t}=-g A \frac{\partial \eta}{\partial x}-\frac{f|U| U P}{A \bullet A} \\
\frac{U_{j}^{n+1}-U_{j}^{n}}{\Delta t}=-g A_{j}^{n+1} \frac{\eta_{j+1 / 2}^{n+1}-\eta_{j-1 / 2}^{n+1}}{\Delta x}-\frac{f\left|U_{j}^{n}\right| U_{j}^{n} P_{j}^{n}}{A_{j}^{n} \bullet A_{j}^{n}}
\end{gathered}
$$

The boundary conditions are

$$
\begin{gathered}
U=0 \text { at } x=L \\
\eta=\eta_{1} \cos (k L) \cos (\omega t) \text { at } x=0
\end{gathered}
$$

Numerical solutions also require initial conditions. The analytical solution to the linear equations with no friction will be used for the initial conditions:

$$
\begin{gathered}
\eta=\eta_{1} \cos (k(x-L)) \text { at } t=0 \\
U=0 \text { at } t=0
\end{gathered}
$$

The stability criterion for the linearized numerical problem is the CFL condition (Roache, 1972):

$$
\frac{\Delta x}{\Delta t}>\sqrt{g h_{\max }}
$$

This condition is met by using $\Delta x=250$ meters and $\Delta t=30$ seconds. Speer(1984) compares this numerical scheme (with a linear form of friction) with Dronker's analytical solution to the linear system with friction. Comparisons between the numerical and analytical show good agreement with the amplitude and phase of elevation.

Time series of elevation, volume flux and velocity of length 5 days are calculated with the above equations. The first day is not included in the analysis to be sure the 
effects of the initial conditions are not biasing the results. The amplitude and phase of the tidal elevation and velocity are determined through least squares harmonic analysis (Boon and Kiley, 1978; Aubrey and Speer, 1985).

\subsubsection{Continuity versus Numerical Solutions}

The continuity method used to determine velocity asymmetry from elevation asymmetry is based on the observations from linear solutions that the elevation at the landward end of the channel determines the velocity asymmetry everywhere in the channel. The validity of the continuity solution for determining the relation between elevation and velocity in the shallow water nonlinear equations is shown by comparisons with numerical solutions.

The velocity asymmetry calculated from the continuity relation is shown through comparisons with numerical solutions to approximate best the velocity asymmetry at the landward half of the channel. The continuity solution determines a phase of the tidal velocity asymmetry that does not vary significantly from the numerical solution throughout the channel (Figure 5). The numerical solution of the amplitude ratio of harmonic to fundamental frequency decreases more than the continuity solution toward the inlet (Figure 6). The amplitude of the fundamental frequency matches the numerical solution on the inlet end of the channel but the amplitude of the harmonic frequency does not decrease as much as the numerical solution toward the inlet (Figure 7). The continuity solution takes into account the nonlinear relation between elevation and velocity at the channel end but the variations in asymmetry down channel as expressed by the continuity solution are similar to the linear solution. The nonlinear variations in velocity down channel as seen in the numerical solution cause a decrease in the amplitude of the harmonic frequency relative to the fundamental which is not accounted for in the continuity solution. 

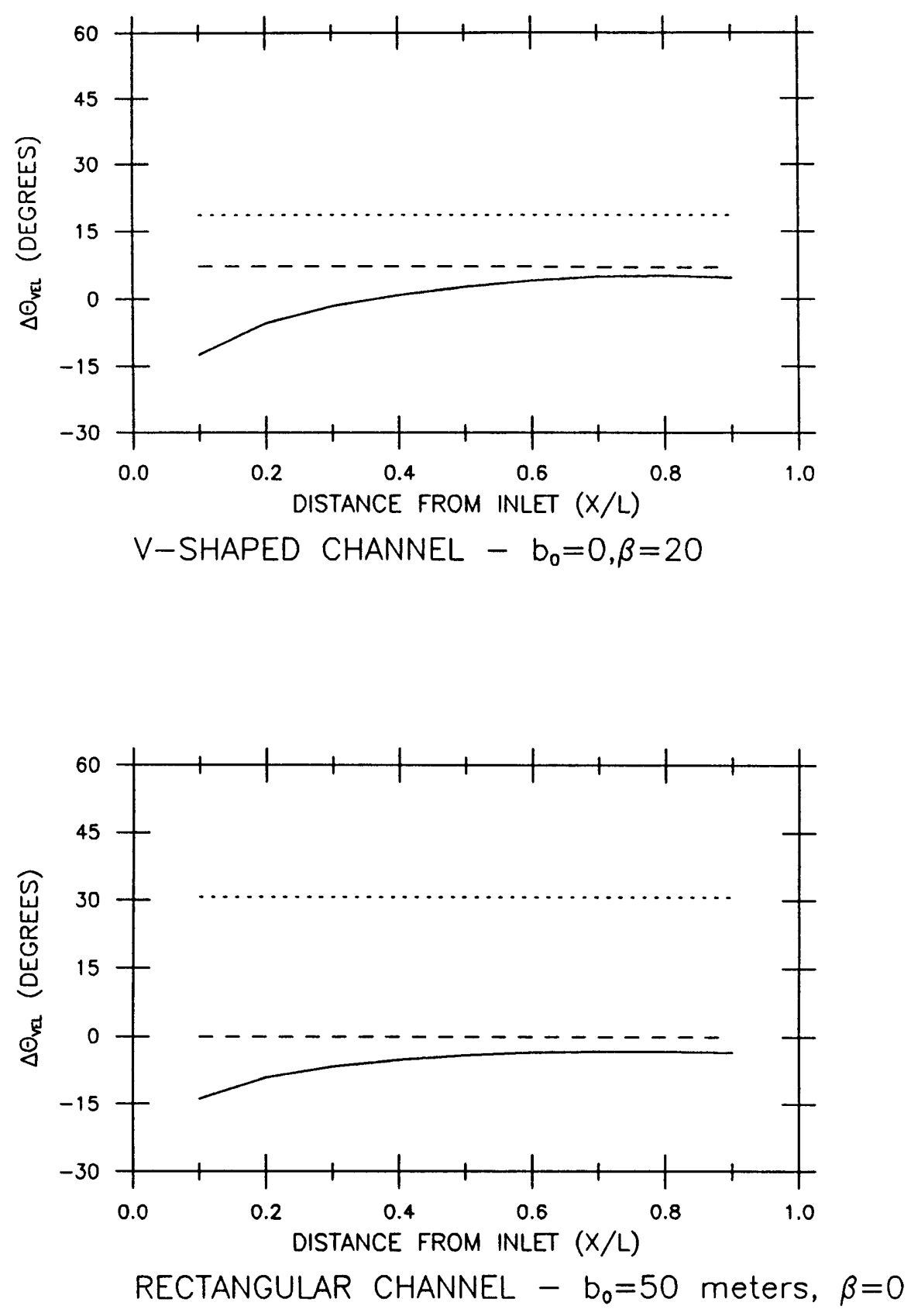

Figure 5: Comparison of the tidal velocity phase differences for the numerical solution (solid), the nonlinear continuity solution (dashed) and the linear solution (dotted) in a rectangular channel and a v-shaped channel $A m p_{F \text { und,inlet,elev }}=0.8 \cos k L$, $A m p_{\text {Harm,inlet,elev }}=0.0, L=10 \mathrm{~km}$., $h=3.0$ meters, $f=.04$, $\left[\Delta \theta_{\text {vel }}=\theta_{2}-2 \theta_{1}\right.$ when $\left.V=V_{1} \cos \left(\omega t-\theta_{1}\right)+V_{2} \cos \left(2 \omega t-\theta_{2}\right)\right]$ 

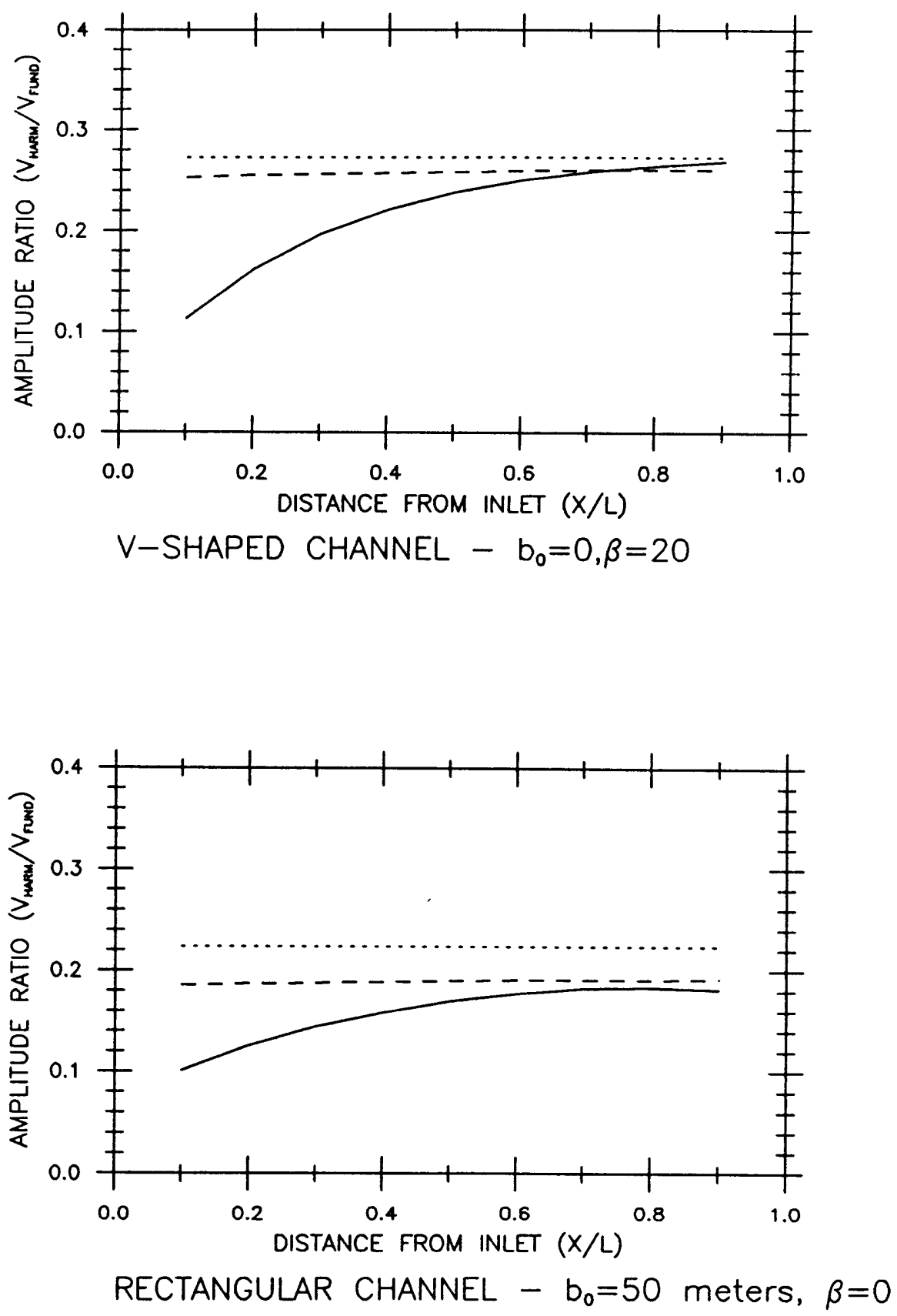

Figure 6: Comparisons of the tidal velocity amplitude ratio for the numerical solution (solid), the nonlinear solution (dashed) and the linear solution (dotted) in a rectangular channel and in a v-shaped channel. Same parameters as previous figure. 

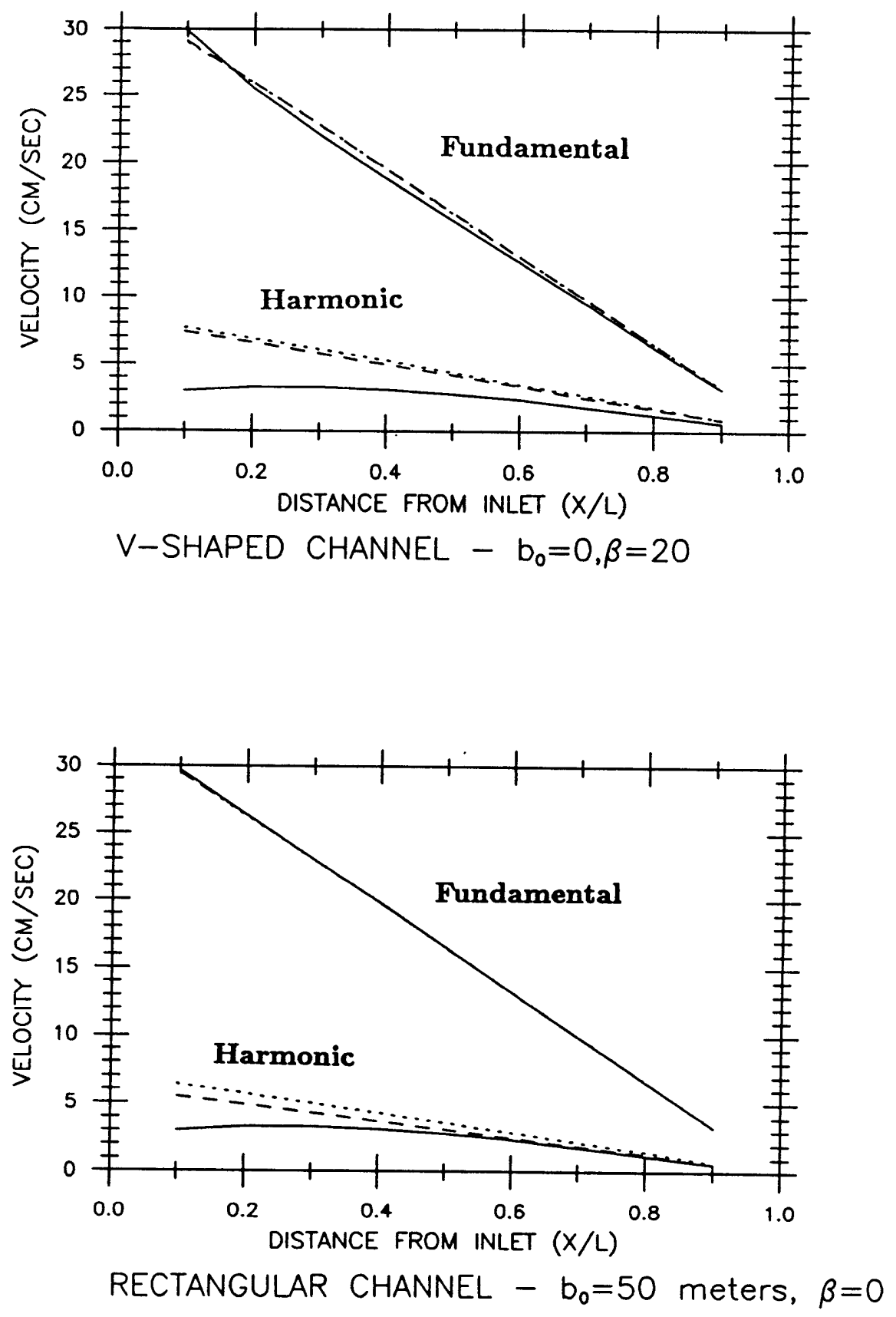

Figure 7: Comparisons of the tidal velocity fundamental and harmonic amplitudes of the numerical solutions (solid), the nonlinear continuity solution (dashed) and the linear solution (dotted). Same parameters as in previous figures. 
The velocity asymmetry determined from numerical solutions decreases towards the inlet as the amplitude ratio of harmonic to fundamental decreases and as the phase difference between fundamental and harmonic $\left(\Delta \theta_{v e l}\right)$ tends away from $0^{\circ}$. This is generally the case for the channel geometries of interest here. If the numerical solutions can be assumed to be the "true solution", the continuity tidal velocity asymmetry is an upper limit for the asymmetry and thus the sediment transport in the inlet.

Numerical solutions show that tidal elevation at both the fundamental and harmonic frequencies reaches an amplitude half way down the channel that stays relatively constant to the end of the channel (Figure 8). Tide gauge data collected from any point in the landward half of the channel should provide the necessary input to determine the velocity asymmetry.

As mentioned previously, rectangular and v-shaped channel geometries are not found commonly in the field. Channel geometries that have flat bottoms and sloping side walls are more likely. Since the relation between velocity and elevation is difficult to determine mathematically for these cases, it is shown numerically that the velocity asymmetry for rectangular channels, calculated through the continuity relation, is also a good approximation for various channel geometries between the rectangular and v-shaped extremes. Figure 9 shows the continuity versus numerical solutions for a channel shape midway between the two extremes. Similar to the rectangular case, the velocity asymmetry at the landward half of the channel is approximated well by the continuity solution. A tidal velocity asymmetry for any channel geometry between a rectangular and v-shaped can be approximated from the continuity solution for a rectangular channel. The solution is valid for the landward half end of the channel and is an upper limit for the asymmetry and thus the sediment transport in the inlet. 

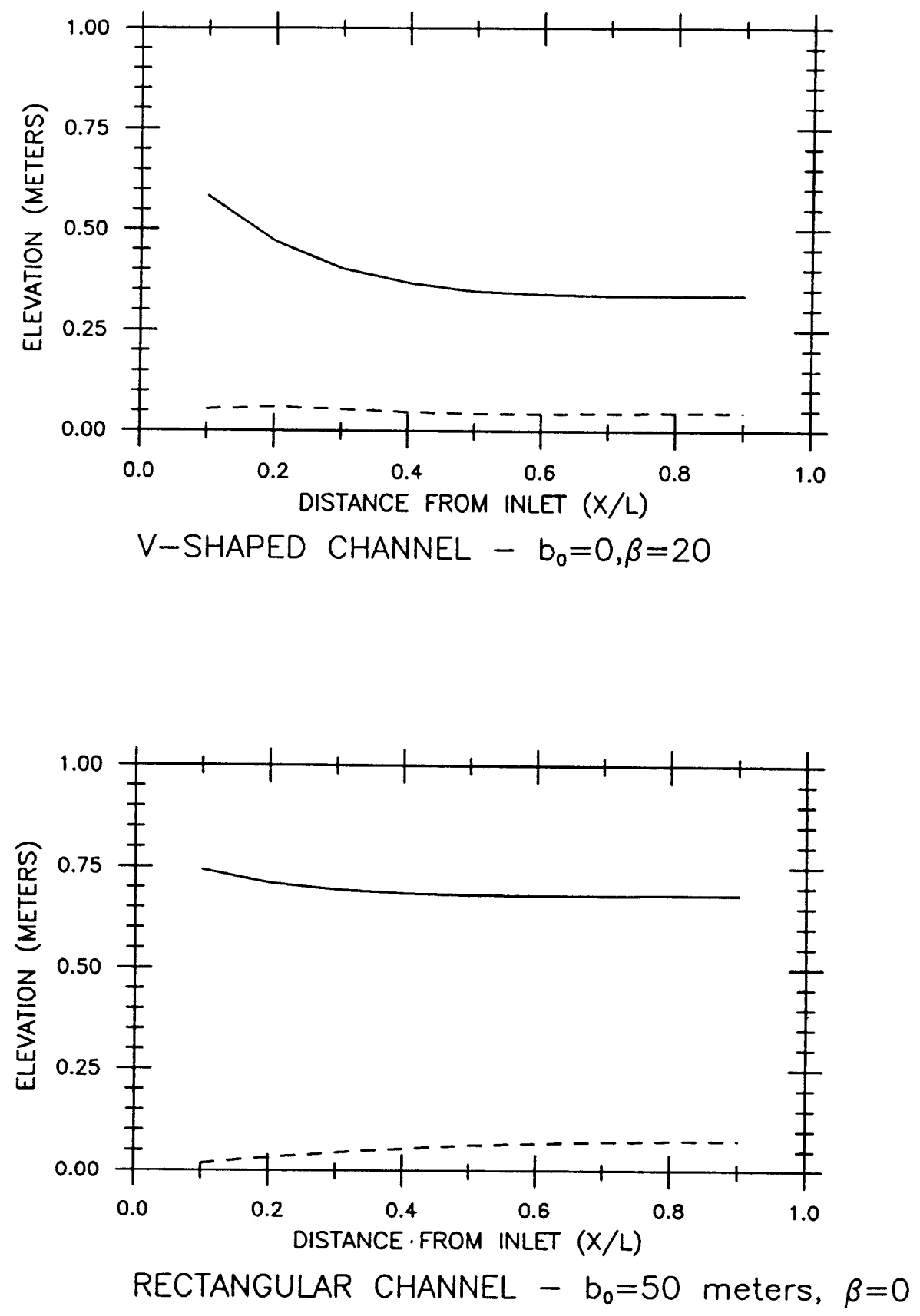

Figure 8: Numerical solution for the elevation amplitude of the fundamental ( $\longrightarrow$ ) and harmonic frequencies (- - ). Same parameters as previous figures. 

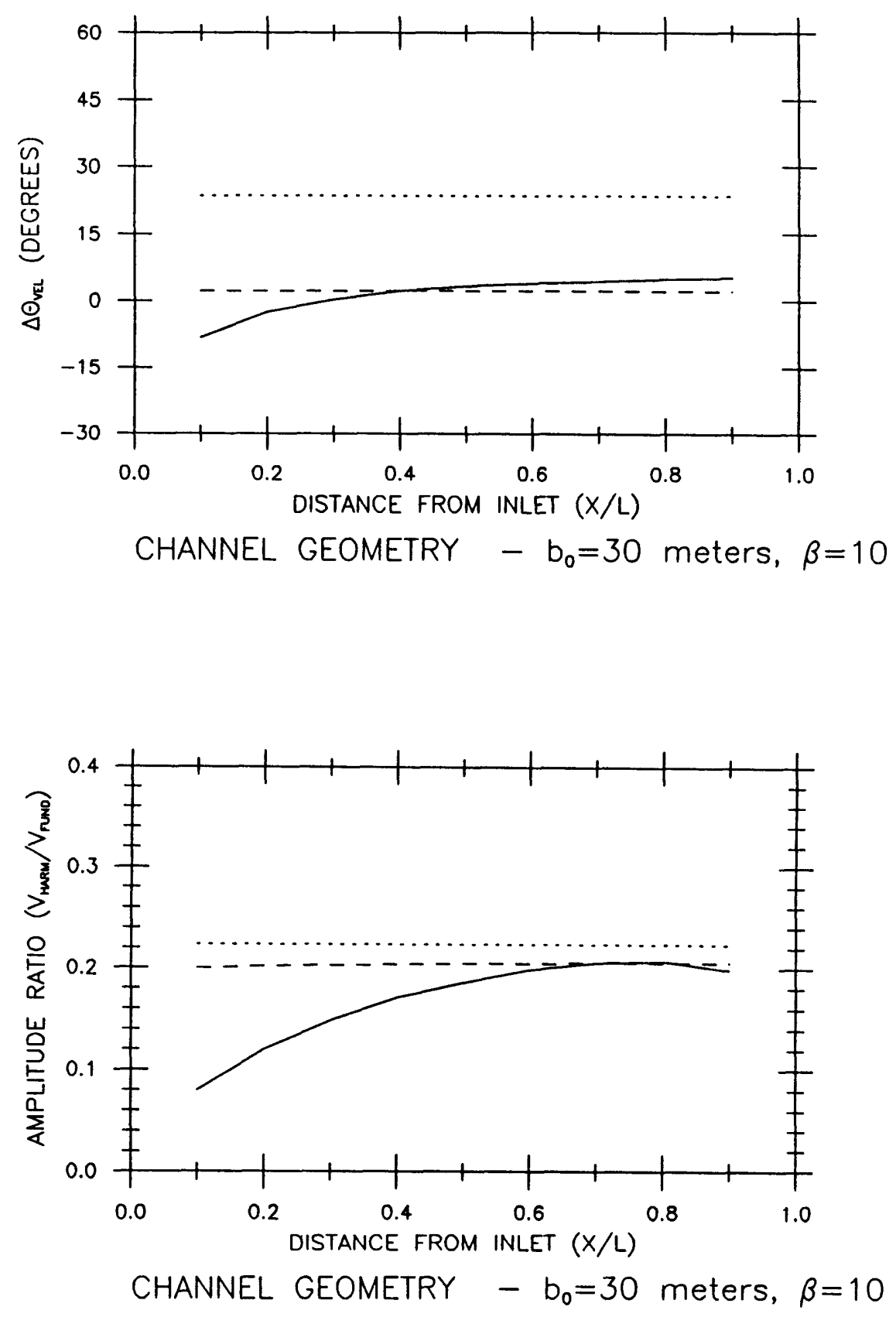

Figure 9: Comparison of the amplitude ratio and phase difference between the numerical solution (solid), the nonlinear continuity solution (dashed) and the linear solution (dotted) for a channel with a flat bottom and sloping side walls. $A m p_{F u n d, \text { inlet,elev }}=0.8 \operatorname{cosk} L, A m p_{\text {Harm, inlet,elev }}=0.0, L=10 \mathrm{~km} ., h=3$ meters, $f=.04, b_{0}=30$ meters, $\beta=10$. 


\section{SEDIMENT TRANSPORT}

Any sediment whose transport equation has a nonlinear relation to velocity may have a net transport in the flood or ebb direction if tidal velocity is composed of a fundamental frequency and its even harmonic. The previous sections have shown how to estimate tidal velocity from tidal elevation data. The purpose of this section is to relate tidal elevation asymmetry to net sediment transport. To determine the degree of net bedload transport due to tidal circulation, the flood-to-ebb ratio can be calculated with a knowledge of the tidal velocity. The sediment parameters cancel out in the ratio allowing a convenient relation independent of sediment type. The flood-to-ebb ratio of suspended load transport cannot be calculated without defining the sediment size and thus is best to calculate separately for the particular system of interest (e.g. Madsen,1975; Smith,1977).

Bedload sediment transport has been expressed in a variety of ways. The MeyerPeter and Müller bedload formula was derived from purely empirical relations (Meyer-Peter and Müller, 1948). The agreement between data and the equation is good. The original equation has been changed by Wilson (1966) to include the Shield's parameter:

$$
q_{s b}=8\left[d \sqrt{\left(\frac{\rho_{g}}{\rho}-1\right) \rho g d}\right]\left(\psi-\psi_{c}\right)^{3 / 2}
$$

where

$q_{s b}$ is volume rate of transport per unit width

$\psi$ is Shield's parameter

$\psi_{c}$ is critical Shield's parameter defining initiation of sediment movement

$d$ is grain diameter

$\rho_{s}$ is density of sediment 
$\rho$ is density of water

$$
\begin{gathered}
\psi=\frac{\tau_{0}}{\left(\frac{\rho_{o}}{\rho}-1\right) \rho g d} \\
\tau_{0}=u_{*}^{2} \rho \\
u_{*} \propto \bar{u} \\
\tau_{0} \propto \bar{u}^{2}
\end{gathered}
$$

where

$\tau_{0}$ is shear stress

$u_{*}$ is shear velocity

$\bar{u}$ is depth averaged velocity

If $\psi_{c} \neq 0$;

$$
q_{a b} \propto\left(\bar{u}^{2}-\bar{u}_{c}^{2}\right)^{3 / 2}
$$

If $\psi_{c}=0$;

$$
q_{a b} \propto \bar{u}^{3}
$$

The Meyer-Peter and Müller equation thus shows that bedload transport is proportional to velocity cubed. The Bagnold equation of bedload transport which is based on the assumption that the volume of transport is proportional to the energy loss per unit area of the bed due to friction between the fluid and the bed is also proportional to velocity cubed (Bagnold, 1963).

Aubrey (1986) calculated the relation between velocity asymmetry and the floodto-ebb ratio using the Meyer-Peter and Müller formula with a $\psi_{c}=0$. Elevation asymmetry for both linear and nonlinear systems now can be related to the flood-toebb ratio of bedload transport using the relationship between elevation and velocity asymmetry as calculated in the previous sections. The Meyer-Peter and Müller equation will be used for this analysis. This information will allow a prediction of 
the ratio of flood-to-ebb bedload transport from knowledge of tidal elevation at the landward end of the channel. The ratio of flood-to-ebb sediment transport is

$$
\frac{\int_{f l o o d}\left(\bar{u}^{2}-\bar{u}_{c}^{2}\right)^{3 / 2} d t}{\int_{e b b}\left(\bar{u}^{2}-\bar{u}_{c}^{2}\right)^{3 / 2} d t}
$$

where $\bar{u}$ is derived from elevation information through Equation 12 (V is equal to $\bar{u})$. The elevation asymmetry of a linear system is plotted versus flood-to-ebb ratio of bedload transport in Figure 10. The linear relation between elevation and velocity is shown by Equations 8 and 9. Phase differences of $0^{\circ}$ and $180^{\circ}$ in elevation will translate into a velocity that will not have a flood or ebb dominance: velocity still may be distorted but will be symmetrical between flood and ebb. The larger the amplitude ratio, the more distorted the velocity signal becomes with potential for greater asymmetry and thus larger differences in the flood and ebb transport.

A nonlinear system shows a similar flood-to-ebb ratio in relation to elevation asymmetry as the linear system (Figure 11). Equations 12 and 13 relate elevation to velocity for a nonlinear system. Results showed earlier that the nonlinear continuity solution for the amplitude ratio and the phase difference were significantly different from the linear solution. The differences between the nonlinear and the linear solutions for the velocity amplitude ratio and the phase difference cancel each other in the calculation of the flood-to-ebb transport ratio. These differences combine to predict a flood-to-ebb ratio that is along the isoline of the flood-to-ebb ratio as calculated in the linear solution. This result can be shown best by Table 1 and Figure 12. Table 1 shows that when $\Delta \theta_{\text {elev }}$ is within $0-90^{\circ}$ (ebb dominant), $\cos \theta$ is positive causing the nonlinear velocity amplitude ratio to be less than that predicted by the linear relation (Equation 12). A decrease in the velocity amplitude ratio by the nonlinear relation causes the system to become less ebb dominant, increasing the flood-to-ebb ratio. The vertical arrow in Figure 12 in the $0-90^{\circ}$ quadrant represents 


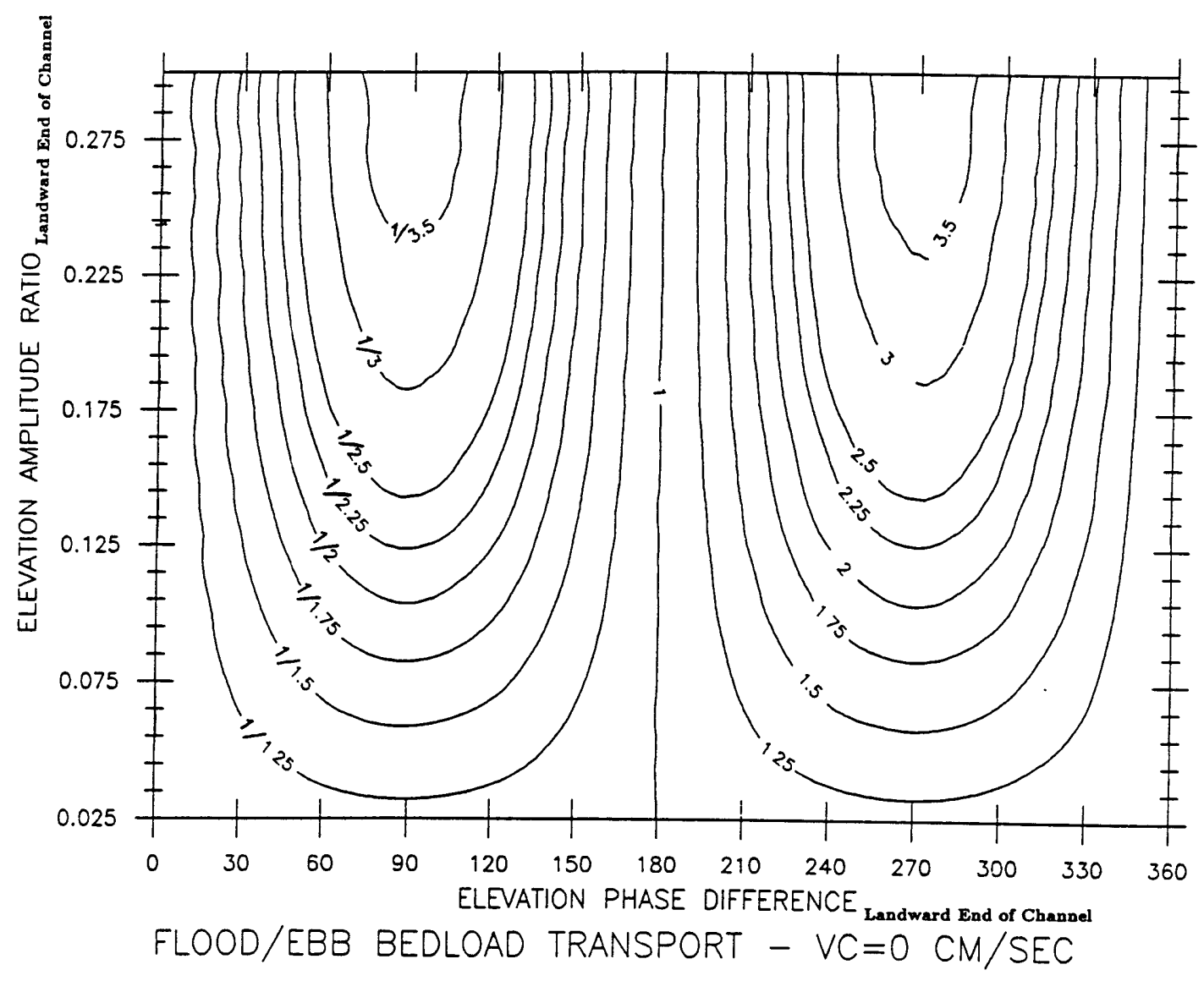

Figure 10: Tidal elevation asymmetry versus flood-to-ebb ratio of bedload sediment transport. Velocity is linearly related to elevation, critical velocity is zero and the relation holds for length of channel if channel length is much less than wavelength of tidal wave. 


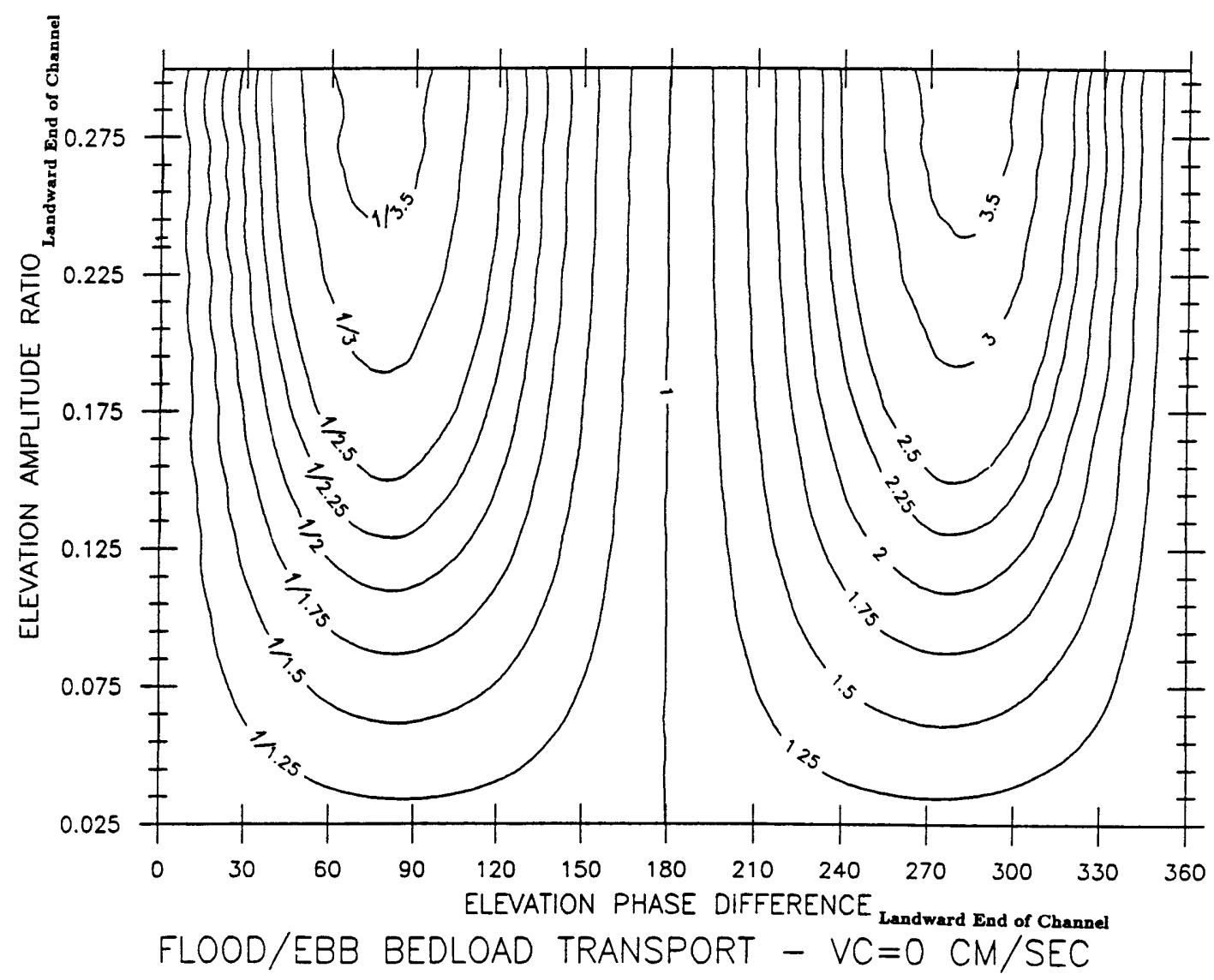

Figure 11: Tidal elevation asymmetry versus flood-to-ebb ratio of bedload sediment transport. Velocity is nonlinearly related to elevation, critical velocity is zero and the relation holds for length of channel if channel length is much less than wavelength of tidal wave. $\eta_{1} / h=0.3$ 


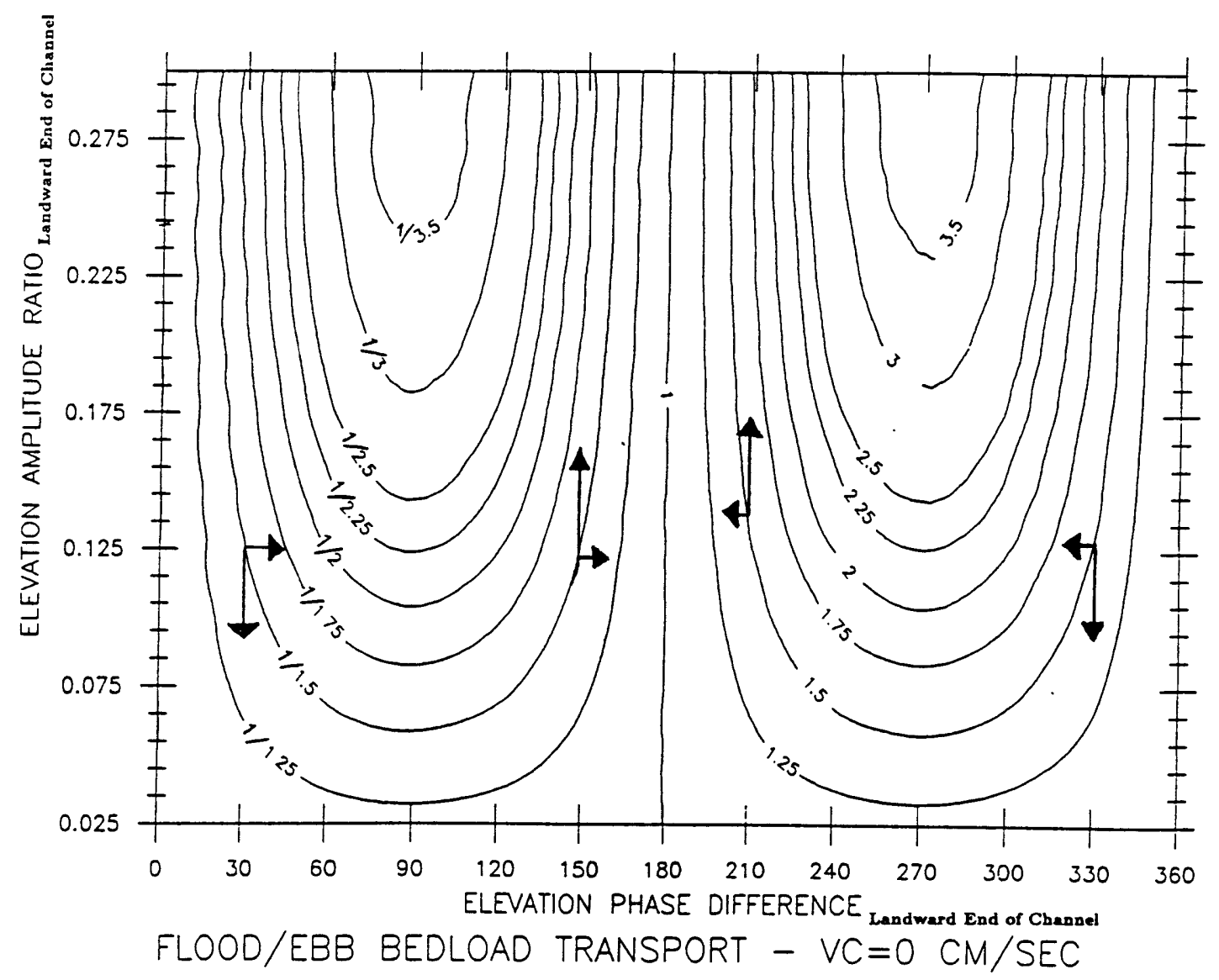

Figure 12: An illustration of how the difference between the nonlinear and the linear solutions for the velocity amplitude ratio and the phase difference cancel each other in the calculation of the flood-to-ebb bedload transport ratio. The vertical arrows show either an increase $\left(0-90^{\circ}, 180-270^{\circ}\right)$ or a decrease $\left(90-180^{\circ}, 270-360^{\circ}\right)$ in the flood-to-ebb ratio due to the nonlinear change in the amplitude ratio. The horizontal arrows show an increase $\left(90-180^{\circ}, 270-360^{\circ}\right)$ or a decrease $\left(0-90^{\circ}, 180-270^{\circ}\right)$ in the flood-to-ebb ratio due to the nonlinear change in the phase difference. Same parameters as Figure 10. 


\begin{tabular}{|c|c|c|c|c|c|c|}
\hline$\Delta \theta_{\text {elev }}$ & $\cos \theta$ & 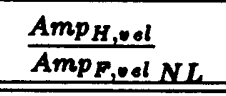 & $\frac{\text { Flood }}{{ }^{*}}$ & $\frac{\Delta \theta_{\text {vell }}}{\Delta \theta_{\text {olev }} N L}$ & $\triangle \theta_{\text {vel }, N L}$ & $\frac{F l o o d}{E b b}{ }^{\dagger}$ \\
\hline $0-90^{\circ}$ & + & $<2 \frac{A m p_{H, \text { slev }}}{A m p_{F, a l e v}}$ & $\uparrow$ & $>90^{\circ}$ & $\rightarrow 180^{\circ}$ & $\downarrow$ \\
\hline $90-180^{\circ}$ & - & $>2 \frac{A m p_{H, \text { slev }}}{A m p_{p_{B}, \text { lev }}}$ & $\downarrow$ & $>90^{\circ}$ & $\rightarrow 0^{\circ}$ & $\uparrow$ \\
\hline $180-270^{\circ}$ & - & 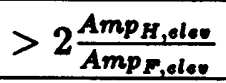 & $\uparrow$ & $<90^{\circ}$ & $\rightarrow 180^{\circ}$ & $\downarrow$ \\
\hline $270-360^{\circ}$ & + & $<2 \frac{A m p_{H, \text { olev }}}{A m p_{F, \text { lev }}}$ & $\downarrow$ & $<90^{\circ}$ & $\rightarrow 0^{\circ}$ & $\uparrow$ \\
\hline
\end{tabular}

Table 1: Differences between the linear and nonlinear relations between elevation and velocity, and their effect on the flood-to-ebb bedload transport ratio. ${ }^{*}$ The change in the linear flood/ebb due to the nonlinear change in the amplitude ratio. $\dagger$ The change in the linear flood/ebb due to the nonlinear change in the phase difference. ( $\mathrm{NL}=$ nonlinear, $\uparrow=$ increases, $\downarrow=$ decreases, $\rightarrow=$ approaches)

the increase in the flood-to-ebb ratio due to the nonlinear velocity amplitude ratio. When $\theta$ is between $0-90^{\circ}$, the phase difference between velocity and elevation is greater than $90^{\circ}$ (Equation 13). $\Delta \theta_{\text {elev }}$ is between $0-90^{\circ}$, thus the $\Delta \theta_{\text {vel }}$ is $90^{\circ+}-180^{\circ+} . \Delta \theta_{v e l}$ gets closer to $180^{\circ}$ due to the nonlinear relation, the system becomes more ebb dominant, and the flood-to-ebb ratio decreases. The horizontal arrow in the $0-90^{\circ}$ quadrangle in Figure 12 represents the decrease in the floodto-ebb ratio due to the nonlinear phase difference. The rest of the quadrants show similar results.

For coarser sediments, the velocity has to reach a certain magnitude before initiation of sediment movement. Figure 13 shows the flood-to-ebb ratio of a linear system with a critical velocity of $20 \mathrm{~cm} / \mathrm{sec}\left(\psi_{c}=.12, u_{*}=1.17\right)$. This critical velocity corresponds to a fine sand with a grain size of $.07 \mathrm{~mm}$. A greater flood-toebb ratio occurs when sediment is stationary for part of the tidal cycle. A nonlinear 


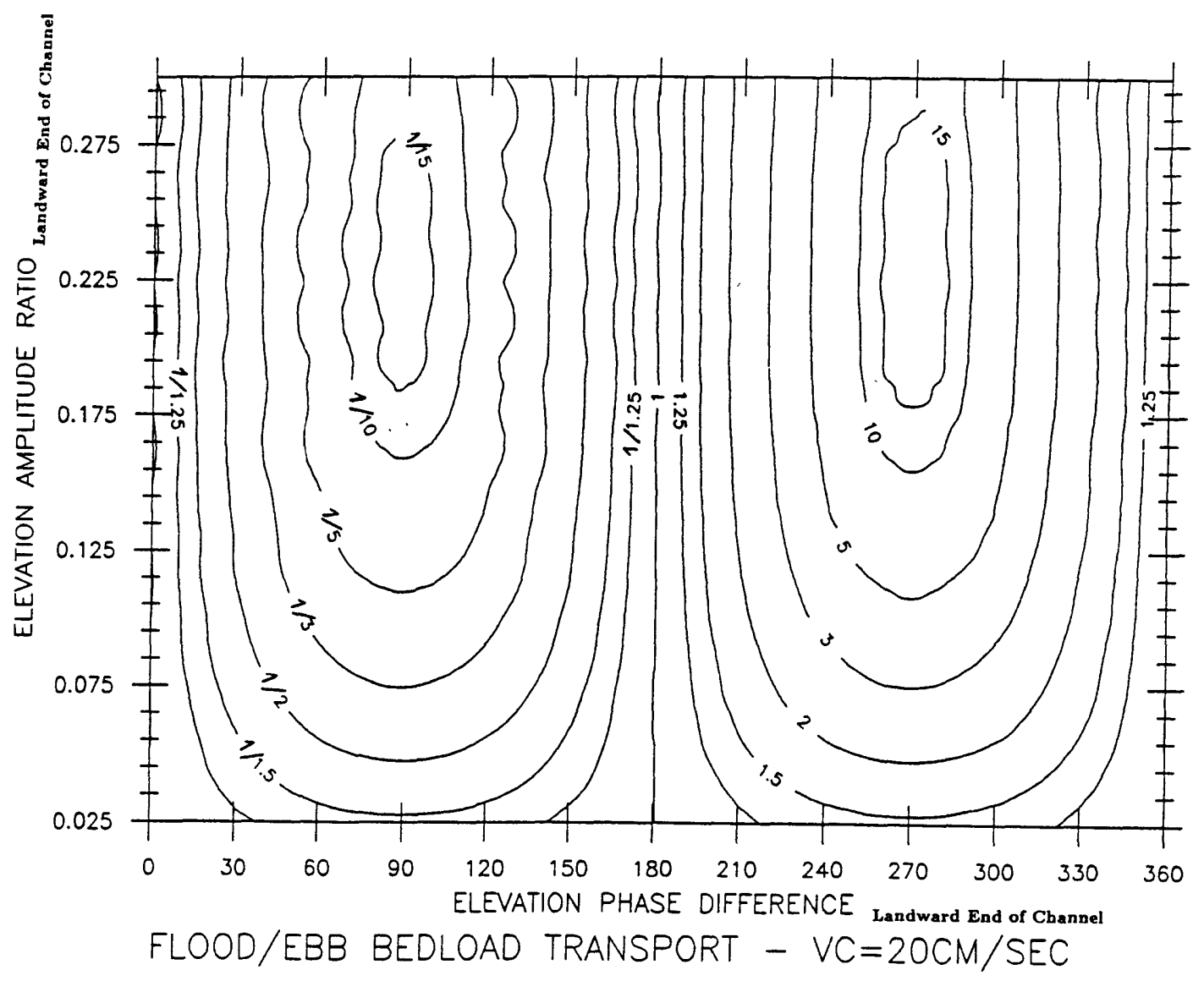

Figure 13: Tidal elevation asymmetry versus flood-to-ebb ratio of bedload sediment transport $2 \mathrm{~km}$ from the inlet. Velocity is linearly related to elevation, critical velocity is $20 \mathrm{~cm} / \mathrm{sec}, L=10 \mathrm{~km}, h=3$ meters, $T_{1}=12 \mathrm{hrs}, T_{2}=6 \mathrm{hrs}$. 
system with a critical velocity of $20 \mathrm{~cm} / \mathrm{sec}$ also shows similar flood-to-ebb ratios as the linear system (Figure 14).

Calculation of the flood-to-ebb ratio of bedload transport for a nonlinear system can be determined from the linear relation. Other sediment transport relations will be related to velocity in various ways which may necessitate the calculation of the nonlinear velocity to determine the sediment transport. The flood-to-ebb transport ratios are calculated here based on the assumption that the continuity solution approximates the true solution adequately. The following section shows the error associated with the continuity relation in terms of the sediment transport.

\section{ERROR DETERMINATION}

The relation between elevation and velocity as determined through the continuity solution of the nonlinear equations does not represent exactly the numerical solution (Figures 5 to 7 ). The continuity solution predicts the nonlinear relation between elevation and velocity at the end of the channel as determined through the numerical solution but the variation in velocity along the channel is similar to the linear solution. The continuity solution does not take into account the nonlinear variation along the channel. Numerical solutions show that most of the nonlinear variation along the channel occurs at the inlet end of the channel.

Estimates of the error in the continuity solution are determined in terms of the difference between the continuity and numerical solutions of the flood-to-ebb ratio of bedload transport. The error in the flood-to-ebb ratio of a nonlinear system is dependent on the parameters of the system and the distance down channel (Figure 15). For channel geometries of interest here, the flood-to-ebb ratio of the continuity solution is similar to or greater than the numerical solution and their dif- 


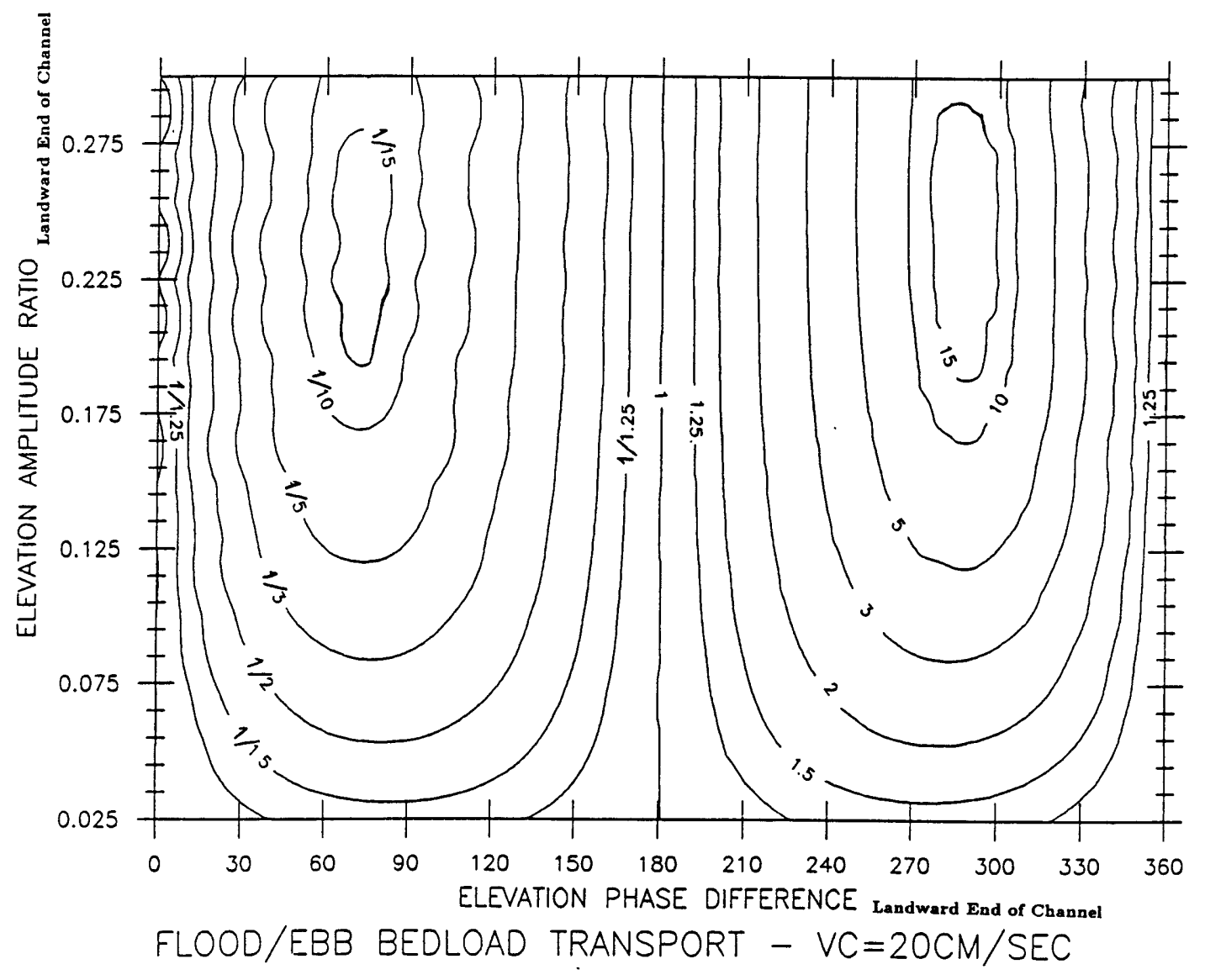

Figure 14: Tidal elevation asymmetry versus flood-to-ebb ratio of bedload sediment transport $2 \mathrm{~km}$ from the inlet. Velocity is nonlinearly related to elevation, critical velocity is $20 \mathrm{~cm} / \mathrm{sec}, L=10 \mathrm{~km}, h=3$ meters, $\eta_{1} / h=0.3, T_{1}=12 \mathrm{hrs}, T_{2}=6 \mathrm{hrs}$. 

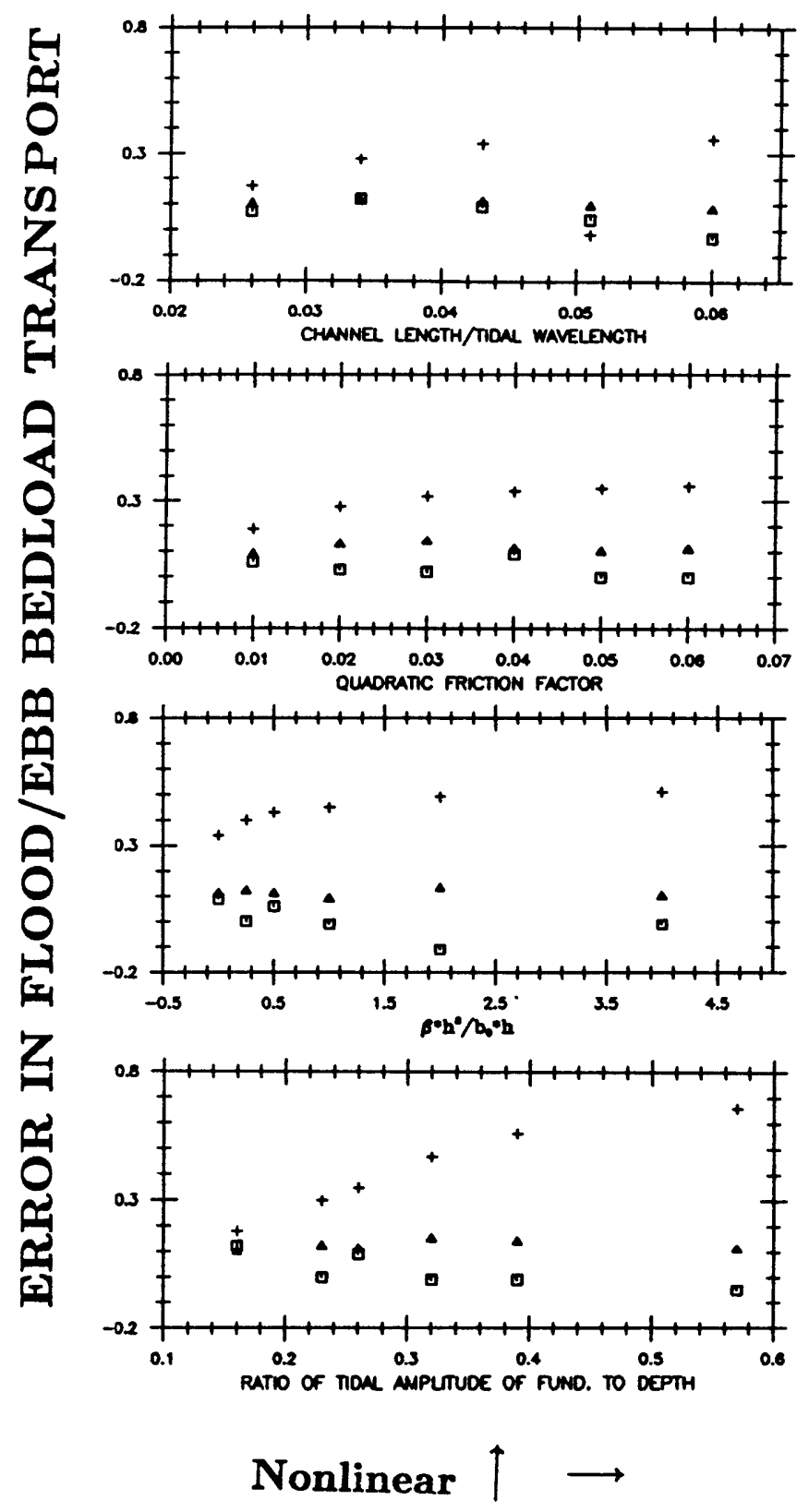

Figure 15: Error determination of the nonlinear continuity solution in terms of the ratio of flood-to-ebb bedload transport as a function of various parameters of the system and distance along channel. $\square=0.8 X / L, \Delta=0.5 X / L,+=0.2 X / L$. Base parameters are $A m p_{\text {fund,inlet, elev }}=0.8 \cos (k L), A m p_{\text {harm, inlet, elev }}=0.0$, $T_{\text {fund }}=12 \mathrm{hrs}, T_{\text {harm }}=6.0, h=3$ meters, $L=10 \mathrm{~km}, b=50$ meters,$\beta=0.0$, $V_{\text {crit }}=0.0$ 
ference increases towards the inlet. This is due to the unpredicted decrease in the amplitude of the harmonic frequency of velocity shown by the numerical solution moving toward the inlet (Figure 7). As the system becomes more nonlinear, the error in the continuity solution at the inlet increases (Figure 15). The difference between the continuity solution and the numerical solution increases towards the inlet and as the system becomes more nonlinear.

\section{CONCLUSIONS}

Net transport of coarse sediment in a coastal embayment is dependent on the differences in circulation between flood and ebb tides. These differences can be quantified by calculating a mean and a measurement for the asymmetry around the mean. $A$ mean circulation caused by wind-driven flow, thermohaline flow or tidal rectification processes may cause a net transport of sediment. But even when the mean circulation is zero, a distortion in the tide causing an asymmetry between flood and ebb may cause a net transport if the sediment transport rate is nonlinearly related to velocity. In a flood or ebb dominant system, the practice of using a tidal residual to determine the net sediment transport neglects transport that is nonlinearly related to velocity.

The type of tidal asymmetry that will cause a net transport is when the tidal velocity is composed of two frequencies, one of which is an even harmonic of the other. Any other combination of frequencies may cause a net transport for a limited interval of time but over the period of the beat frequency, net transport will be zero. The extent of the velocity asymmetry between flood and ebb is measured by the amplitude ratio of the harmonic frequency to fundamental frequency and their phase

difference. The closer the amplitude ratio is to 1.0 and $\Delta \theta_{v e l}$ is to $0^{\circ}$ or $180^{\circ}$, the 
greater the asymmetry.

Tidal velocity asymmetries can be estimated from tidal elevation data. The relationship between elevation asymmetry and velocity asymmetry in a linear system (Equations 8 and 9 ) is determined from the equations of motion of the fluid. In a nonlinear system, the equations do not have an exact solution. The relation between elevation and velocity in a nonlinear system is found through the continuity equation (Equations 12 and 13). The differences between the nonlinear estimate of velocity amplitude ratio and phase difference and the linear estimate are significant in shallow water systems (amplitude of tide/depth of water $\gg 0$ ).

Finite difference numerical solutions of the one dimensional, shallow water nonlinear equations are compared to the continuity relation. The continuity relation is in excellent agreement with numerical solutions at the landward end of the channel, however, the variation along the channel in a nonlinear system as represented by the numerical solution differs from the continuity relation increasingly toward the inlet. Numerical solutions show the greatest velocity asymmetry at the landward end of the channel and the least at the inlet end. The greatest variation in asymmetry along the channel (velocity and sea surface) concentrates at the inlet end. The more nonlinear the system, the greater the difference between the continuity relation and numerical solutions at the inlet.

Calculation of the flood-to-ebb ratio for bedload transport and its relation to elevation asymmetry is similar whether the linear or nonlinear relation between elevation and velocity is used. The differences between the nonlinear and the linear solutions of the amplitude ratio and the phase difference cancel each other causing a similar flood to ebb ratio as predicted by the linear relation. Sediment whose transport rates are related to velocity in ways other than velocity cubed may need to take the nonlinear variations into account when calculating the flood to ebb ratio. 
A combination of velocity asymmetry, maximum velocity, and the critical velocity of the sediment will cause a variation in the net transport along the channel. Velocity asymmetries for the channel geometries shown in the previous sections decrease towards the inlet causing the ratio of flood-to-ebb net transport to decrease towards the inlet. In a channel with one end closed, maximum velocity increases towards the inlet. If velocity asymmetry (flood-to-ebb transport ratio) and critical velocity were constant along channel, a greater volume of transport would occur toward the inlet due to the increase in velocity. A depositional area in the channel will occur where there is a decrease in the volume of sediment transported. This could take place when velocity decreases toward the end of the channel more than the increase in the velocity asymmetry. If the velocity asymmetry increases toward the channel end more than the decrease in the maximum velocity, an erosional area could occur. Sediment type may vary along the channel and bedforms may change, consequently, the critical velocity may vary. Determination of the net transport into or out of the embayment as well as depositional and erosional areas within the channel should consider these three mechanisms that affect the sediment transport. Lateral effects as well as bathymetric variations in the channel may also be important in determining the sediment transport.

The number of parameters involved in trying to generalize the sediment transport in a coastal embayment is prohibitive. Each system must be looked at separately. The emphasis of this analysis is to determine from tidal elevation data whether, for the particular system of interest, there will be a net transport of sediment due to tidal asymmetries and in what direction. Estimates of the transport of sediment from field data or theoretical analysis should take into consideration the possibility of net transport due to tidal asymmetries. 


\section{References}

Aubrey, D.G., 1986. Hydrodynamic controls on sediment transport in well-mixed bays and estuaries. Physics of Shallow Estuaries and Bays, J. van de Kreeke (ed.), Springer-Verlag, 245-258.

Aubrey, D.G. and P.E. Speer, 1985. A study of non-linear tidal propagation in shallow inlet/estuarine systems. Part I: Observations. Estuarine, Coastal and Shelf Science, 21, 185-205.

Bagnold, P.A., 1963. Mechanics of marine sedimentation. In: M.N. Hill (Editor), The Sea, 3. Wiley, New York, N.Y., 507-582.

Boon, J.D., III and K.P. Kiley, 1978. Harmonic analysis and tidal prediction by the method of least squares. Spec. Report No. 186, Virginia Institute of Marine Science, Gloucester Pt., VA. 49 pp.

Cloern, J.E. and F.H. Nichols, 1985. Time scales and mechanisms of estuarine variability, a synthesis from studies of San Francisco Bay. Hydrobiologia, 129, 229-237.

Dronkers, J.J., 1964. Tidal Computations in Rivers and Coastal Waters. North Holland Publishing Company, Amsterdam, 516 pp.

Friedrichs, C.T. and D.G. Aubrey, submitted. Nonlinear tidal distortion in shallow well-mixed estuaries: A synthesis. Estuarine, Coastal and Shelf Science.

Gallagher, B.S. and W.H. Munk, 1971. Tides in shallow water: Spectroscopy. Tellus, 23, 346-363.

Kabbaj, A. and C. LeProvost, 1980. Nonlinear tidal waves in channels: A perturbation method adapted to the importance of quadratic bottom friction. Tellus, 32, 143-163.

Kreiss, H., 1957. Some remarks about nonlinear oscillations in tidal channels. Tellus, 9, 53-68.

Madsen, O.S., 1975. Lecture Notes on Mechanics of Sediment Transport in Steady Flow, MIT, Dept. of Civil Engr., 47-64.

Meyer-Peter, E. and R. Müller, 1948. Formulas for bedload transport. Proc. 2nd Meet., Mtl. Assoc. Hydraulic Struct. Res., Append. 2: 39-64.

Pingree R.D. and L. Maddock, 1978. The $M_{4}$ tide in the English Channel derived from a nonlinear numerical model of the $M_{2}$ tide. Deep Sea Research, 26, 53-68.

Prandle, D., 1980. Modeling of tidal barrier schemes: an analysis of the openboundary problem by reference to $\mathrm{AC}$ circuit theory. Estuarine and Coastal Marine Science, 11, 53-71. 
Pritchard, D.W., 1971. Two-dimensional Models, Chap. 11-2 of Estuarine Modelling: An Assessment (Edited by G.H. Ward and W.H. Esprey), Water Quality Office, Environmental Protection Agency. NTIS Publication No. PB206-807.

Roache, P.J., 1972. Computational Fluid Dynamics, Hermosa Publishers, Albuquerque, $434 \mathrm{pp}$.

Smith, J.D., 1977. Modeling of Sediment Transport on Continental Shelves, The Sea, Vol. 6, (Edited by E.D. Goldberg, I.N. McCave, J.J. O'Brien, \& J.H. Steele), John Wiley \& Sons, NY 539-577.

Speer, P.E. and D.G. Aubrey, 1985. A study of non-linear tidal propagation in shallow inlet/estuarine systems. Part II: Theory. Estuarine, Coastal and Shelf Science, 21, 207-224.

Speer, P.E., 1984. Tidal distortion in shallow estuaries. Ph.D. thesis, WHOI-MIT Joint Program in Oceanography, Woods Hole, MA. 210pp.

Tee, K.T., 1976. Tide-induced residual current, a 2-D nonlinear numerical tidal model, Journal of Marine Research, 34, 603-628.

Uncles, R.J., 1981. A note on tidal asymmetry in the Severn estuary. Est. Coast. Mar. Sci., v. 13, 419-431.

Wilson, K.C., 1966. Bed-load transport at high shear stress. Proc. ASCE, J. Hydraul. Div., 92 (HY6): 49-65.

Zimmerman, J.T.F., 1981. Dynamics, diffusion and geomorphological significance of tidal residual eddies. Nature, 290, 549-555. 


\section{Appendix 1}

Summary of the relationship between tidal elevation and velocity.

Tidal elevation can be represented as a summation of cosine functions with varying amplitudes and phases.

$$
\eta=A_{1} \cos \left(\omega t-\theta_{1}\right)+A_{2} \cos \left(2 \omega t-\theta_{2}\right)+\ldots
$$

A harmonic analysis of the tidal elevation will provide the amplitudes $\left(A_{1}, A_{2}, \ldots\right)$ and phases $\left(\theta_{1}, \theta_{2}\right)$ of the tidal constituents. If the elevation can be represented as a combination of a fundamental frequency and its even harmonic, net sediment transport can occur. The degree of asymmetry of the tidal velocity in a channel and thus the sediment transport can be determined from the tidal elevation at the landward half end of the channel.

The velocity asymmetry in a channel is represented by the following equations. The amplitude ratio of the first harmonic $\left(H_{1}\right)$ to the fundamental $(\mathrm{F})$ is

$$
\frac{A m p_{v e l, H_{1}}}{A m p_{v e l, F}}=\frac{\sin (2 k(x-L))}{\sin (k(x-L))}\left[\left(\frac{A_{2}}{A_{1}} \cos \theta-\frac{A_{1}}{4 h}\right)^{2}+\left(\frac{A_{2}}{A_{1}} \sin \theta\right)^{2}\right]^{1 / 2} .
$$

The phase difference in the velocity is

$$
\triangle \theta_{v e l}=\arctan \left(\frac{\frac{A_{2}}{A_{1}} \cos \theta-\frac{A_{1}}{4 h}}{-\frac{A_{2}}{A_{1}} \sin \theta}\right)+0^{\circ} \text { or } 180^{\circ} .
$$

Choose $0^{\circ}$ or $180^{\circ}$ so $\Delta \theta_{\text {vel }} \approx \Delta \theta_{\text {elev }}+90^{\circ}$. The tidal elevation parameters are the following:

$A_{1}$ is the tidal elevation amplitude of the fundamental frequency at the landward half end of the channel

$A_{2}$ is the tidal elevation amplitude of the first harmonic frequency at the landward half end of the channel 
$\theta$ is the phase difference between the fundamental and harmonic frequencies of the tidal elevation at the landward half end of the channel. $\theta=\Delta \theta_{\text {elev }}=\theta_{2}-2 \theta_{1}$

$L$ is the length of the channel

$\mathrm{h}$ is the mean water depth 